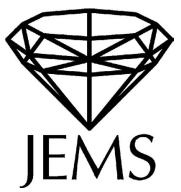

Gerd Faltings

\title{
Algebraic loop groups and moduli spaces of bundles
}

Received March 15, 2001 / final version received April 11, 2002

Published online November 19, 2002 - (c) Springer-Verlag \& EMS 2002

Abstract. We study algebraic loop groups and affine Grassmannians in positive characteristic. The main results are normality of Schubert-varieties, the construction of line-bundles on the affine Grassmannian, and the proof that they induce line-bundles on the moduli-stack of torsors.

\section{Introduction}

The present note aims to explain some basic facts about algebraic loop groups. Some are well known in characteristic zero, but not so in positive characteristics, and the paper started as an attempt to check them in full generality. The topics are

- construction of loop groups and their central extensions,

- the infinite Grassmannians and flag spaces,

- the theory of Schubert varieties,

- integrable representations,

- moduli spaces and line-bundles on them,

- the Suguwara construction.

Most of the facts are neither surprising nor difficult to prove. Maybe the Suguwara construction in positive characteristics comes as a little surprise, because its usual definition involves complicated denominators. It should be mentioned that normality of Schubert varieties has been shown (at about the same time as the research for our paper) for the groups $S L_{n}$ by Pappas and Rapoport, using a different method ([12]). Also I learned that P. Littelmann gave a general proof ([9]). In general there seems to be some confusion because several definitions of "Schubert varieties" seem possible. Although logically it suffices to use ours I have tried to explain the relation to others as well as I can understand it. I thank V. Drinfeld for useful comments.

\section{Construction of loop groups}

Suppose $G$ is a linear algebraic group defined over $\mathbb{Z}$ (or over any other base). For an indeterminate $t R((t))$ denotes the ring of Laurent-series.

G. Faltings: Max-Planck-Institut für Mathematik, Vivatsgasse 7, 53111 Bonn, Germany 


\section{Definition 1}

- The positive loop group $L^{+} G$ is the affine group scheme representing the functor

$$
L^{+} G(R)=G(R[[t]]) .
$$

- The strict positive loop group $L^{++}(G) \subset L^{+}(G)$ is the kernel of the reduction map to $G$.

- The negative loop group $L^{-} G$ is the ind-affine group scheme representing

$$
L^{-} G(R)=G\left(R\left[t^{-1}\right]\right) .
$$

- $L^{--}(G) \subseteq L^{-} G$ is again the kernel of reduction.

- $L G$ is the ind-affine group with

$$
L G(R)=G(R((t)) .
$$

That these functors are indeed representable (or, for $L^{-} G, L^{--} G, L G$, indrepresentable) by affine schemes is easily seen (see also [1, Prop. 1.2]). For example for $H=S L_{d} L H(R)$ is the union of its subfunctors $L_{n} H(R)$ consising of matrices of determinant 1

$$
A=\sum_{i \geq-n} A_{i} \cdot t^{i},
$$

with the $A_{i} d \times d$ matrices with coefficients in $R$. Obviously $L_{n} H$ is representable by a closed subscheme of infinite affine space, and the inclusion $L_{n} H \subseteq L_{n+1} H$ is a closed immersion. Also

$$
L_{m}(H) \cdot L_{n}(H) \subseteq L_{m+n} H
$$

and $\left(L_{n} H\right)^{-1} \subseteq L_{n(d-1)} H$. These assertions also hold for proper closed $G \subseteq$ $H=S L_{d}$.

Now recall the construction of the infinite Grassmannian. Consider (for $H=$ $S L_{d}$ ) the functor $\mathcal{D}_{H}$ which associates to a ring $R$ the set $\mathcal{D}_{H}(R)$ consisting of projective $R[[t]]$-modules $L \subset R((t))^{d}$ with

1. $t^{n} \cdot R[[t]]^{d} \subseteq L \subseteq t^{-n} \cdot R[[t]]^{d}$ for $n \gg 0$

2. $\operatorname{det}(L)=R[[t]]$.

$D_{H}$ is an ind-algebraic scheme, increasing union of the closed subschemes where the value of $n$ in the first condition above is fixed. Also it admits a natural action by $L H=L S L_{d}$. This action is transitive (locally in the Zariski-topology of $\operatorname{Spec}(R)$ any $L$ is free and thus a transform of $R[[t]]^{d}$ ), and the stabiliser of the origin $R[[t]]^{d}$ is $L^{+} H$.

One defines on $\mathcal{D}_{H}$ a very ample line bundle $\mathcal{L}=\operatorname{det}_{R}(L)^{\otimes-1}$. In fact what is naturally defined is the "difference"

$$
\operatorname{det}_{R}\left(L_{1}\right) \otimes \operatorname{det}_{R}\left(L_{2}\right)^{\otimes-1} .
$$

For example choose an $n$ with $t^{n} \cdot R[[t]]^{d} \subseteq L_{1,2}$ and consider the quotient of the $R$-determinants of the quotients $L_{1,2} / t^{n} \cdot R[[t]]^{d}$. It is independant of $n$. 
Fixing $L_{1}$ and varying $L_{2}=L$ then defines $\mathcal{L}$. Because of this procedure the action of $L H$ extends only to a projective action on $\mathcal{L}$. However the stabiliser of a fixed lattice (like $L^{+}(H)$ ) acts honestly on $\mathcal{L}$.

Dually this also holds for $L^{-} H$ : Namely consider the submodule $M=t^{-1}$. $R\left[t^{-1}\right]^{d} \subset R((t))^{d}$. Then $\operatorname{det}_{R}(L)^{\otimes-1}$ is equal to the determinant of cohomology of the complex

$$
0 \rightarrow L \oplus M \rightarrow R((t))^{d} \rightarrow 0
$$

or more precisely to the determinant of the quotient complex obtained by dividing both terms by

$$
t^{n} \cdot R[[t]]^{d} \oplus M .
$$

Hence an action of the stabiliser $L^{-} H$ of $M$. Also the actual determinant of the map above (i.e. of the quotient) defines a section

$$
\vartheta_{M} \in \Gamma\left(\mathcal{D}_{H}, \mathcal{L}\right)
$$

invariant under $L^{-} H$. Namely choose an integer $n$ such that $L$ contains $t^{n} \cdot R[[t]]^{d}$. Then the determinant of cohomology is equal to that of the complex

$$
L / t^{n} \cdot R[[t]]^{d} \rightarrow R((t))^{d} /\left(t^{n} \cdot R[[t]]^{d} \oplus M\right),
$$

and $\vartheta_{M}$ is the determinant of the boundary map in this complex.

(One can show that its transforms under $L H$ define a projective embedding, but we do not need that.)

Now consider the complement $\mathcal{D}_{H}-\Theta$ of the zero-set $\Theta$ of $\vartheta_{M}$.

Lemma 2 The action of $L^{--} H$ on the origin $L_{0}=R[[t]]^{d}$ defines an isomorphism

$$
L^{--} H \stackrel{\sim}{\rightarrow} \mathcal{D}_{H}-\Theta .
$$

Proof Suppose $L \in \mathcal{D}_{H}(R)$ lies in the complement of $\Theta$, that is $L \oplus M=R((t))^{d}$. If $\left\{e_{1}, \ldots, e_{d}\right\}$ denotes the standard base of $L_{0}=R[[t]]^{d}$, then there exists a unique $d \times d$-matrix

$$
h \in 1+t^{-1} \cdot R\left[t^{-1}\right]^{d \times d}
$$

with $h\left(e_{i}\right) \in L$. We claim that these elements generate $L$, that is that $L=h\left(L_{0}\right)$ :

Namely choose a large integer $n$ with $t^{n} \cdot h \in R[t]^{d \times d}$ integral and such that $t^{n} \cdot L_{0} \subseteq t \cdot L$. Then it suffices that the $h\left(e_{i}\right)$ generate $L / t^{n} \cdot L_{0}$ as $R[[t]]$-module. Each element in this quotient can be represented by an element $f \in L \subseteq R((t))^{d}=$ $L_{0} \oplus M$ such that its projection to $L_{0}$ is a linear combination of $t^{j} \cdot e_{i}$ with $j<n$. However the projection of $h\left(t^{j} \cdot e_{i}\right)$ is of the form $t^{j} \cdot e_{i}$ modulo terms with lower $t$-powers. Thus by decreasing induction one can make the $L_{0}$-projection of $f$ and thus also $f$ itself vanish.

Now as $L$ and $L_{0}$ have the same $R[[t]]$-determinant the determinant of $h$ must be a unit in $R[[t]]$. Especially it contains no negative $t$-powers, thus it is 1 and $h \in L^{--} H$. It is clear that the map

$$
h: \mathcal{D}_{H}-\Theta \rightarrow L^{--} H
$$

is an inverse to the $L^{--} H$-action on $L_{0}$. 
It follows from the lemma that the multiplication map

$$
L^{--} H \times L^{+} H \rightarrow L H
$$

is an open immersion. We claim that this also holds for certain subgroups.

Corollary 3 Suppose $G \subseteq H$ is a closed subgroup, which is the stabiliser of an element $e \in E$ for an algebraic representation of $H=S L_{d}$ on a finitely generated projective $R$-module E. (For example this holds if $G$ is defined and flat over the integers $\mathbb{Z}$, and admits no non trivial characters $G \rightarrow \mathbb{G}_{m}$.)

Then the multiplication map

$$
L^{--} G \times L^{+} G \rightarrow L G
$$

is an open immersion. An element $g \in L G(R)$ lies in the image iff $g\left(L_{0}\right) \in \mathcal{D}_{H}-\Theta$.

Proof We obviously must show that if a product $g=g_{-} \cdot g_{+}$with $g_{-} \in L^{--} H(R)$, $g_{+} \in L^{+} H(R)$ lies in $L G(R)$, then also both factors are in $L G$. But $g_{-}^{-1}(e)=$ $g_{+}(e) \in E((t))$ lies both in $e+t^{-1} \cdot E\left[t^{-1}\right]$ and in $E[[t]]$, thus must be equal to $e$.

As a consequence one easily constructs the quotient $\mathcal{D}_{G}=L G / L^{+} G$ : It is an ind-scheme which has an open covering by translates of $L^{--} G$. Also the map $\mathcal{D}_{G} \rightarrow \mathcal{D}_{H}$ is a closed immersion. The orbits of $L^{-} G$ on $\mathcal{D}_{G}$ form an interesting "stratification with strata of finite codimension". As this is not used we refrain from elucidating what this should mean. However for completeness we list the orbits in a special case.

Namely assume that $G$ is a connected split reductive algebraic group, and $T \subseteq G$ a split maximal torus, $Y=\operatorname{Hom}\left(\mathbb{G}_{m}, T\right)$ its group of one parameter subgroups. Any $\rho \in Y$ defines an element $\rho(t) \in L T \subseteq L G$. Also our base ring $R$ is a field $k$.

Lemma 4 We have a disjoint union

$$
L G(k)=\bigcup_{\rho \in Y} L^{-} G(k) \rho(t) L^{+} G(k) .
$$

Proof Any $g \in L G(k)$ defines a $G$-torsor $P$ on $\mathbb{P}_{k}^{1}$ by gluing trivial torsors on $\mathbb{P}^{1}-\{\infty\}=\mathbb{A}^{1}$ and on the formal completion along $\{\infty\}$. The assertion amounts to the fact that this torsor reduces to the $T$-torsor defined by some $\rho \in Y$, or that it just reduces to a $T$-torsor.

We know that the associated group-scheme $G_{P}$ contains a Borel, since this is true over $\mathbb{A}^{1}$ and the space of Borels is proper over $\mathbb{P}^{1}$. If we divide the Borel by its uniponent radical we obtain a torus, isomorphic to the constant torus $T$. The choice of a Borel reduces the structur group of $P$ to the standard Borel $B \subset G$, and by pushout we obtain a $T$-torsor. This torsor is given by a one parameter group $\rho \in Y$. We claim that we can chose these data such that $\alpha(\rho) \geq 0$ for any positive root (occuring in the unipotent radical).

Namely it suffices to do this for simple roots. Number these as $\left\{\alpha_{1}, \ldots, \alpha_{l}\right\}$, and denote by $\epsilon_{i}$ the dual weights (that is dual weights on the maximal torus of the semisimple part, and trivial on the connected center). Some multiples of them are 
weights of $T$, so some power of the associated line-bundle $\mathcal{L}\left(\epsilon_{i}\right)$ is defined on $\mathbb{P}^{1}$, and we can define its degree ( a rational number) by linearity.

We define an operation which for a simple root $\alpha_{i}$ makes the degree of $\mathcal{L}\left(\alpha_{i}\right)$ positive, while not changing the degrees of $\mathcal{L}\left(\epsilon_{j}\right)$ for $j \neq i$. If we reply it repeatedly as long as some $\mathcal{L}\left(\alpha_{i}\right)$ has negative degree, we increase in each step the degree of some $\mathcal{L}\left(\epsilon_{i}\right)$ by a positive integer. However the line-bundle $\mathcal{L}\left(2 \sum_{i} \epsilon_{i}\right)$ is a subbundle of the vectorbundle $\mathcal{E}$ associated to the $G$-representation of highest weight $2 \sum_{i} \epsilon_{i}$, thus has its degree bounded independantly of our choice of a Borel. Thus the process must stop.

If for such a simple $\alpha_{i} \alpha_{i}(\rho)$ is negative, we remedy this by change of Borels, without affecting the other $\epsilon_{j}(\rho)$ 's. Namely in $G^{a d}$ we have a parabolic $P_{i}$ (associated to $\alpha_{i}$ ) and a quotient $P_{\alpha} \rightarrow P G L_{2}$. The associated $P G L_{2}$-torsor is an Azumaya-algebra of the form $\mathcal{E} n d(\mathcal{E})$ for a vectorbundle $\mathcal{E}$ on $\mathbb{P}^{1}$ of rank 2. The Borel in there corresponds to a quotient line bundle $\mathcal{L}$, and the degree $\alpha_{i}(\rho)$ is the difference of the degree of the kernel and that of $\mathcal{L}$, that is

$$
\alpha_{i}(\rho)=\operatorname{deg}(\mathcal{E})-2 \cdot \operatorname{deg}(\mathcal{L}) .
$$

If this is negative we write $\mathcal{E}=\mathcal{O}(a) \oplus \mathcal{O}(b)$ with $a \leq b$, and replace $\mathcal{L}$ by the quotient $\mathcal{O}(a)$. Its normaliser defines the new Borel in $G_{P}$. As some power of $\mathcal{L}\left(\epsilon_{j}\right)$ $(j \neq i)$ is defined by a character of $P_{i}$ which is not changed by our operation, its degree remains the same.

Thus assume that $\rho$ is dominant. Then the unipotent radical of the Borel (in $G_{P}$ ) has a filtration, with successive quotients isomorphic to twisted additive groups, namely to $\mathcal{O}(\alpha(\rho)), \alpha$ running through the positive roots. As these have trivial $H^{1}$ the same is true for the whole unipotent radical. This means that the fibre over $\rho$ of the pushforward

$$
H^{1}\left(\mathbb{P}^{1}, B\right) \rightarrow H^{1}\left(\mathbb{P}^{1}, T\right)=Y
$$

is trivial, and our torsor is indeed defined by $\rho$.

Similarly one can consider double classes for the subgroups

$$
\mathcal{B}^{-} \subset L^{-} G, \mathcal{B}^{+} \subseteq L^{+} G
$$

which are the preimages of the Borels $B^{ \pm} \subset G$. Now the double classes are indexed by $Y \propto W$, the semidirect product of $Y$ and the Weyl-group. If $\mathcal{N}^{-} \subset \mathcal{B}^{-}$denotes the preimage of the unipotent radical $N^{-} \subset B^{-}$, then the map

$$
\mathcal{N}^{-} \times \mathcal{B}^{+} \rightarrow L G
$$

is an open immersion.

Thus we define the affine flag-variety

$$
\mathcal{X}_{G}=L G / \mathcal{B}^{+} \text {. }
$$

The $\mathcal{N}^{-}$-orbit trough the origin $*$ is an open subset of $\mathcal{X}_{G}$ isomorphic to $\mathcal{N}^{-}$.

For the group $H=S L_{d} \mathcal{X}_{H}$ classifies $R[[t]]$-lattices $L \subset R((t))^{d}$ with determinant $R[[t]]$, together with a complete flag in $L / t \cdot L$ :

$$
F_{0}=t \cdot L \subset F_{1} \subset \ldots \subset F_{d}=L .
$$


It admits $L^{+} H$ - and $L^{-} H$-equivariant line-bundles $\mathcal{L}_{i}=\operatorname{det}_{R}\left(F_{i}\right)^{\otimes-1}$. Furthermore if we choose a complete flag in $M=R\left[t^{-1}\right]^{d}$

$$
G^{0}=M \supset G^{1} \supset \ldots \supset G^{d}=t^{-1} \cdot M,
$$

we obtain global sections

$$
\vartheta_{i}=\operatorname{det}\left(F_{i} \rightarrow R((t))^{d} / G^{i}\right) \in \Gamma\left(\mathcal{X}_{G}, \mathcal{L}_{i}\right) .
$$

These are invariant under $\mathcal{N}^{-}, T$ acts on them via the inverse of the i-th fundamental character $\epsilon_{i}$, and they generate $\mathcal{L}_{i}$ on the "big cell" $\mathcal{N}^{-} *$. By pullback via $G \rightarrow H$ we obtain such line-bundles and sections also over $\mathcal{X}_{G}$.

\section{Geometry of orbits}

From now on we assume that $G$ is split, simply connected, semi-simple and simple. That is $G$ is the Chevalley group associated to an irreducible root system $\Phi$, and $Y$ is generated by the coroots $H_{\alpha}$, with $\alpha$ running through the simple roots $\Delta \subseteq \Phi^{+}$. Unless otherwise mentioned we choose $R=\mathbb{Z}$ as basering. Let $\theta \in \Phi^{+}$denote the highest root.

We denote affine roots by $n+\alpha$, with the corresponding root-vector $t^{n} \cdot X_{\alpha}$ in the Lie-algebra $L(g)=\mathfrak{g}((t))$. Such a root is positive if either $n>0$, or $n=0$ and $\alpha \in \Phi^{+}$, otherwise negative. Thus the Lie-algebra of $\mathcal{N}^{-}$is generated by root-vectors belonging to negative roots. Also number the simple roots of $G$ as $\Delta=\left\{\alpha_{1}, \ldots, \alpha_{l}\right\}$, and denote by $\alpha_{0}=1-\theta$ the remaining affine simple root. For each affine simple root we have an associated inclusion $S L_{2} \subset L G$ which factors over a parabolic (or "parahoric"?) group $\mathcal{P}_{i} \subset L G$. that is $\mathcal{P}_{i}$ contains the Borel $\mathcal{B}^{+}$. We denote by $U_{i}^{+} \subset \mathcal{B}^{+}$the corresponding unipotent subgroup ( $\cong \mathbb{G}_{a}$ ), by $U_{i}^{-} \subset \mathcal{N}^{-}$its opposite. By conjugation with $W^{\text {aff }}$ we obtain an $U_{\alpha}$ for any affine root $\alpha$.

The affine Weyl-group $W^{a f f}$ is a Coxeter group generated by the reflections $\left\{s_{i} \mid 0 \leq i \leq l\right\}$. For each $w \in W^{\text {aff }}$ its length $l(w)$ is the minimal number of factors in a product of $s_{i}$ 's representing $w$. Any such product realising the minimum is called a reduced decomposition of $w$.

The Bruhat-order on $W^{\text {aff }}$ is defined as follows: Suppose $w=s_{1} \cdot s_{2} \cdots s_{r}$ is a reduced decomposition. Then the elements $w^{\prime} \leq w$ are obtained by replacing some factors by 1 (after that one has to remove further pairs of factors to get a minimal representation). It is known that this is independant of the choice of reduced decomposition.

Now for a moment we work over an algebraically closed field $k$. It is known that $W^{\text {aff }}$ parametrises the double classes of $\mathcal{B}^{+}$in $L G$ (Bruhat decomposition), that is the $\mathcal{B}^{+}$-orbits in $\mathcal{X}_{G}$. We have seen that the same holds for $\mathcal{N}^{-}$-orbits. If we order orbits by inclusions of closures, this order will be the Bruhat-ordering respectively its inverse. To prove this we need some preparation:

Definition 5 For $w \in W^{\text {aff }}$ the Schubert-variety $C(w) \subset \mathcal{X}_{G}$ is the closure of $\mathcal{B}^{+} w *$. 
To describe $C(w)$ recall that for each reduced decomposition $w=s_{1} \cdot s_{2} \cdots s_{r}$ we obtain a $\mathcal{B}^{+}$-equivariant (Demazure) resolution

$$
D(w) \rightarrow C(w)
$$

(The notation does not quite reflect the complexity of the situation). It is defined as

$$
D(w)=\mathcal{P}_{1} \times{ }^{\mathcal{B}^{+}} \mathcal{P}_{2} \times \mathcal{B}^{+} \cdots \times{ }^{\mathcal{B}^{+}} \mathcal{P}_{r} / \mathcal{B}
$$

Here " $\times \mathcal{B}^{+}$" denotes the contraction. If $w=w_{1} \cdot s_{i}$ then $D(w)$ is a fiber-bundle over $D\left(w_{1}\right)$ with fibre $\mathcal{P}_{i} / \mathcal{B}^{+}=\mathbb{P}^{1}$. Thus by induction it is smooth, proper, and irreducible. We usually define subschemes of $D(w)$, or more generally schemes mapping to $D(w)$, by giving conditions on the "factors" (which lie in $\mathcal{P}_{i}$ ).

Also $D(w)$ has a stratification where closed strata are defined by requiring that the some factors lie in $\mathcal{B}^{+}$. The image in $\mathcal{X}_{G}$ of such a closed stratum contains the element $w^{\prime} \in W^{a f f}$ which is the product of the remaining $s_{i}$ 's. Also from the theory of Tits-systems (see for example [11, II]) one knows that only such elements of $W^{\text {aff }}$ can lie in the image. The open stratum maps isomorphically onto $\mathcal{B}^{+} w *$. From this follows the assertion about the ordering on $\mathcal{B}^{+}$-orbits, as well as the independance of the Bruhat-order from the choice of reduced decomposition.

To go further we need some notation: For an element $w \in W^{\text {aff }}$ define subgroups $\mathcal{N}_{w}^{-} \subseteq \mathcal{N}^{-}, \mathcal{N}_{w}^{+} \subseteq \mathcal{N}^{+}$as

$$
\begin{aligned}
& \mathcal{N}_{w}^{-}=\mathcal{N}^{-} \cap w \mathcal{N}^{-} w^{-1}, \\
& \mathcal{N}_{w}^{+}=\mathcal{N}^{+} \cap w \mathcal{N}^{-} w^{-1} .
\end{aligned}
$$

One easily shows that

$$
\mathcal{N}^{-} \cong \mathcal{N}_{w}^{-} \times \prod U_{\alpha}
$$

where the product is over the negative affine roots $\alpha$ for which $w^{-1}(\alpha)$ is positive. Also $\mathcal{N}_{w}^{+}$is the product of the their $w$-conjugates $w^{-1} U_{\alpha} w$.

For example choose a strictly dominant one-parameter group $\rho \in Y$, and choose a very large positive constant $c$. This defines a linear form on affine roots by the rule

$$
l(\alpha)=l((m+\beta))=m \cdot c+\beta(\rho) .
$$

Thus $\alpha$ is positive iff $l(\alpha)$ is. Now $\mathcal{N}^{-}$is obtained from $\mathcal{N}_{w}^{-}$by adding affine roots $\alpha$ with $l(\alpha)<0, l\left(w^{-1}(\alpha)\right)>0$. Order these by the value $l(\alpha)$, and multiply from the right (with $U_{\alpha}$ ) in that order.

For later use we also need the subgroup $\mathcal{N}^{-}(n) \subset \mathcal{N}^{-}$whose $R$-points are elements in $L^{-} G(R)=G\left(R\left[t^{-1}\right]\right)$ which lie modulo $t^{-n}$ in $L^{-} T$. Here $n$ should be big enough so that $\mathcal{N}^{-}(n) \subseteq \mathcal{N}_{w}^{-}$. One then finds again

$$
\mathcal{N}_{w}^{-} \cong \mathcal{N}^{-}(n) \times \prod U_{\alpha}^{-},
$$

where the product is over the negative affine roots $\alpha=(-m+\beta)$ which occur in $\mathcal{N}_{w}^{-}$but not in $\mathcal{N}^{-}(n)$. 
Also one checks that

$$
w \mathcal{N}^{-} * \cong \mathcal{N}_{w}^{-} \times \mathcal{N}_{w}^{+} w *
$$

Next we claim that the closure of an $\mathcal{N}^{-}$-orbit $\mathcal{N}^{-} w^{\prime} *$ meets $C(w)$ iff it contains $w$ iff $w \geq w^{\prime}$ :

Firstly if $w \geq w^{\prime}$ consider the the first factor $s_{i}$ missing in $w^{\prime}$. Consider the affine line in $D(w)$ which is $U_{i}^{-}$in the corresponding factor, and which has the same coordinates as $W^{\prime}$ in all other positions. This maps to the $\mathcal{N}^{-}$-orbit through $w^{\prime} *$ and contains a bigger $w^{\prime \prime} \leq w$ in its closure. Continue by induction.

Conversely assume the Bruhat cell $C(w)$ meets the $\mathcal{N}^{-}$-orbit through $w^{\prime}$. There exists a $\mathbb{G}_{m}$-action on $\mathcal{X}_{G}$ which contracts $\mathcal{N}^{-}$and fixes all $w *$ : It is the product of a strictly dominant one-parameter group $\rho: \mathbb{G}_{m} \rightarrow G$, and a power of the $\mathbb{G}_{m}$-action on $L G$ which scales $t$ (Virasoro-action via $L_{0}$ ), as in the linear form $l$ used above. On the one hand this action respects the Bruhat-cell $C(w)$, on the other the limit of an element in $\mathcal{N}^{-} w^{\prime} *$ is $w^{\prime}$. Thus $w^{\prime} \leq w$, which is already close to the converse.

To derive this converse completely assume that $C(w)$ meets the closure of the $\mathcal{N}^{-}$-orbit through $w^{\prime} *$. We may replace $w$ by a smaller element and assume that the closure meets $\mathcal{B}^{+} w *$. However this is contained in the open $\mathcal{N}^{-}$-invariant set

$$
\mathcal{N}^{-} w \mathcal{N}^{-} *=\mathcal{N}^{-} \mathcal{N}_{w}^{+} w *=\mathcal{N}^{-} \mathcal{B}^{+} w *
$$

which must contain $w^{\prime}$, and we already know that this means $w^{\prime} \leq w$.

Finally this result implies that the order on $\mathcal{N}^{-}$-orbits is indeed the inverse Bruhat-order.

Next choose a finite set of $w \in W^{a f f}$. We assume that it contains with each $w$ also any $w^{\prime} \leq w$. This implies that the set

$$
\Omega=\bigcup w \mathcal{N}^{-} * \subset \mathcal{X}_{G}
$$

is open and $\mathcal{N}^{-}$-stable. We can chose an integer $n$ such that for each chosen $w$ and each negative affine $\operatorname{root} \alpha=(-m+\beta)(\beta \in \Phi)$ with $m \geq n w^{-1}(\alpha)$ is also negative, that is $\mathcal{N}^{-}(n) \subseteq \mathcal{N}_{w}^{-}$.

Then

Lemma 6 There exists an open covering of $\Omega$ by $\mathcal{N}^{-}(n)$-invariant open subsets equivariantly isomorphic to

$$
\mathcal{N}^{-}(n) \times \mathbb{A}^{r}
$$

Especially the quotient

$$
\mathcal{N}^{-}(n) \backslash \Omega
$$

exists, is smooth, and covered by affine spaces.

Proof We have

$$
w \mathcal{N}^{-} * \cong \mathcal{N}_{w}^{-} \cdot \mathcal{N}_{w}^{+} w * \cong \mathcal{N}^{-}(n) \times \mathbb{A}^{a} \times \mathbb{A}^{b}
$$


What this lemma tells us is that our $\mathcal{X}_{G}$ behaves very much like usual flag varieties. To wit:

Theorem 7 - Choose $\Omega, \mathcal{N}^{-}(n)$ as before. The $\mathcal{N}^{-}$-orbits in $\Omega$ are pullbacks of locally closed subschemes of $\mathcal{N}^{-}(n) \backslash \Omega$ isomorphic to affine spaces.

- These orbits are parametrised by the chosen set of w's. The codimension of the w-orbit is $l(w)$. Especially the orbits of codimension one correspond to the elementary reflections $s_{i}$. Call the corresponding orbit $\Theta_{i}$.

- There exist on $\mathcal{X}_{G}$ line bundles $\mathcal{L}_{i}(0 \leq i \leq l)$. These are equivariant under $L^{+} G$ and $L^{-} G$. They have a global section $\vartheta_{i}$ invariant under $\mathcal{N}^{-}$, with divisor $\Theta_{i}$, and transforming under $T$ with the inverse of the fundamental character $\epsilon_{i}(1 \leq i \leq l)$ respectively trivially $(i=0)$.

- For each $i$ consider the embedding

$$
\mathbb{P}^{1}=\mathcal{P}_{i} / \mathcal{B}^{+} \rightarrow \mathcal{X}_{G}
$$

The degree of the pullback of $\mathcal{L}_{i}$ to it is 1 if $i=j, 0$ else.

Proof The images in $\mathcal{N}^{-}(n) \backslash \Omega$ of orbits are isomorphic to $\mathcal{N}^{-}(n) \backslash w \mathcal{N}_{w}^{-}$, thus affine spaces of codimension $l(w)$. Furthermore the $\mathcal{N}^{-}$-action on $\Omega$ is described by the groupoid

$$
\mathcal{N}^{-} \times \Omega \rightarrow \Omega \times \Omega \text {. }
$$

Dividing by $\mathcal{N}^{-}(n)$ gives the smooth groupoid

$$
\mathcal{N}^{-}(n) \backslash \mathcal{N}^{-} \times^{\mathcal{N}^{-}(n)} \Omega \rightarrow \mathcal{N}^{-}(n) \backslash \Omega \times \mathcal{N}^{-}(n) \backslash \Omega .
$$

As the orbits are stable under it so are their closures, and they define line-bundles $\mathcal{L}_{i}$ with sections $\vartheta_{i}$ equivariant with respect to the groupoid. That means we get $\mathcal{N}^{-}$-equivariant such objects on $\Omega$. As $\Omega$ can be made arbitrarily big we obtain them on all of $\mathcal{X}_{G}$.

To compute the degree of $\mathcal{L}_{i}$ on the $j$-th $\mathbb{P}^{1}$, we first note that for $i \neq j$ the projective line does not meet $\Theta_{i}$, so the degree is zero. For $i=j$ the intersection is transversal and the result is 1 :

Let $\mathbb{P}^{1} \subset \mathcal{X}_{G}$ denote the projective line corresponding to $S L_{i}$. To compute the intersection multiplicity with $\Theta_{i}$ can use the open set

$$
\Omega=\mathcal{N}^{-} \cdot \mathbb{P}^{1} \cong \mathcal{N}_{s_{i}}^{-} \times \mathbb{P}^{1}
$$

Then $\Theta_{i}=\mathcal{N}_{s_{i}}^{-} \times\{\infty\}$ has degree 1 .

For equivariance under $L^{+} G$ we use that $\mathcal{X}_{G}$ is the inductive limit of Schubertcells $C(w)$ which are flat, geometrically normal, and irreducible over $\mathbb{Z}$ and have a $\mathcal{B}^{+}$-equivariant resolution $D(w) \rightarrow C(w)$. Especially over each field $k$

$$
\Gamma(C(w) \otimes k, \mathcal{O})=\Gamma(D(w) \otimes k, \mathcal{O})=k .
$$

This is shown in the next section, of course without making use of our present theorem. Furthermore one knows that line-bundles on $D(w)$ are characterised by their degrees on certain $\mathcal{B}^{+}$-invariant lines $\mathbb{P}^{1}$. By direct image this also holds for line-bundles on $C(w)$, and the lines in question are the $\mathbb{P}^{1}$ 's corresponding to 
simple affine roots $\tilde{\alpha}_{i}$ with $s_{i} \leq w$ (see [11, Ch. 12, Prop. 6]). Thus a line-bundle on $C(w)$ is uniquely determined by the degree of its restriction to these lines.

A cofinal system of $C(w)^{\text {'s }}$ is $G$-invariant and thus $L^{+} G$-invariant. As the degrees on lines $\mathbb{P}^{1}$ must be invariant $\left(L^{+} G\right.$ is connected) any line-bundle on these $C(w)$ is equivariant under a central extension of $L^{+} G$ and thus (uniquely) under $L^{+} G$ as $G$ is simply connected. Passing to the inductive limit it follows that any line-bundle on $\mathcal{X}_{G}$ is $L^{+} G$-equivariant.

Finally the actions of $G$ and $\mathcal{N}^{-}$on $\mathcal{L}_{i}$ must coincide on the intersection $N^{-}$ because they can only differ by a character which must be trivial on that unipotent group. Thus equivariance under $L^{-} G$.

Remark. In fact these actions are induced from that of a central $\mathbb{G}_{m}$-extension $\tilde{L} G$ of $L G$. This follows because $L^{+}(G)$ and $L^{-}(G)$ generate $L G$ (as sheaf in the Zariski-topology), and because over each algebraically closed field $\mathrm{k}$ all global sections $\Gamma\left(\mathcal{X}_{G} \otimes k, \mathcal{O}\right)=k$ are constant. Also note that equivariance is already known for the line-bundles obtained by pullback via representations $G \rightarrow H=$ $S L_{d}$ (which will generate a subgroup of finite index in the Picard-group).

Remark. An alternative construction of the $\mathcal{L}_{i}$ uses that the stack $L^{-} G \backslash \mathcal{X}_{G}$ is the moduli stack of $G$-bundles on $\mathbb{P}^{1}$, and the construction of line-bundles on this moduli-stack, which however is not necessarily easier than our geometric approach.

Also for the construction of the $\mathcal{L}_{i}$ we could have replaced the groups $\mathcal{N}^{-}(n)$ by the congruence subgroups of $L^{-} G$, that is those elements which are the identity modulo $t^{-n}$.

\section{Normality of Schubert-varieties}

The main result is

Theorem 8 Each $C(w)$ is normal, Cohen-Macaulay, and has rational singularities. Also they form an ind-scheme (using the Bruhat order) isomorphic to $\mathcal{X}_{G}$.

Remark. In characteristic zero this is completely shown in [8]. We review these facts below after first establishing some properties of the Schubert-cells.

In positive characteristics most of it is in [11, Ch. 18]. However as I could not quite follow the proof of Lemma 134 (perhaps because of deficiencies in my French), I repeat the arguments. Note that in [11] there is no problem about normality of Schubert cells because the flag variety itself is defined as the inductive limit of normalised Schubert cells (in our terminology) $\tilde{C}(w)$. What is new here is that $C(w)$ itself is normal. Also Littelmann gives a representation-theoretic proof for normality. It is a little bit difficult to decide which definition of "Schubert-cell" he uses, but it seems to be ours.

Begin of Proof. We assume that $k$ has positive characteristics $p>0$. By [11, Lemma 135] the variety $D(w)$ is Frobenius-split, compatible with the inclusions $D\left(w^{\prime}\right) \subset D(w)$ for $w^{\prime}<w$ : Namely the splitting is given by a global section of the 
$(p-1)$-st power of the determinant of the logarithmic tangent bundle (vectorfields tangential to the boundary). However this determinant is the pullback of a linebundle on $\mathcal{X}_{G}$ which is just the product of all $\mathcal{L}_{i}$. As this product is generated near the origin by the product of $\theta_{i}$ 's we get a splitting.

Denote by $\tilde{C}(w)$ the normalisation of $C(w)$, so that $\pi_{w}: D(w) \rightarrow C(w)$ factors as

$$
D(w) \rightarrow \tilde{C}(w) \rightarrow C(w) .
$$

The first map is a Stein-map, the second finite and birational. Furthermore the inclusions $D\left(w^{\prime}\right) \subset D(w)$ for $w^{\prime}<w$ define maps $\tilde{C}\left(w^{\prime}\right) \rightarrow \tilde{C}(w)$.

We have the following result, combining Lemmas 32, 136, and Assertion 6 in Th. 5. of [11]:

Lemma 9 a) All higher direct images $R^{i} \pi_{w, *}\left(\mathcal{O}_{D(w)}\right)$ vanish, and the fibres of $\pi_{w}$ are geometrically connected.

b) The normalisation $\tilde{C}(w) \rightarrow C(w)$ is a universal homeomorphism.

c) For $w^{\prime}<w$ the map

$$
\tilde{C}\left(w^{\prime}\right) \rightarrow \tilde{C}(w)
$$

is a closed immersion.

Proof Use induction over $l(w)$. If $w=s_{i} \cdot w^{\prime}$ with $l\left(w^{\prime}\right)=l(w)-1$ we can factor $\pi_{w}$ as

$$
D(w)=\mathcal{P}_{i} \times{ }^{\mathcal{B}} D\left(w^{\prime}\right) \rightarrow \mathcal{P}_{i} \times{ }^{\mathcal{B}} C\left(w^{\prime}\right) \rightarrow C(w) .
$$

The first map satisfies a) by induction. For the second consider the reduced $\mathcal{P}_{i}$-stable subscheme

$$
Z=\bigcup C\left(w^{\prime \prime}\right) \subset C\left(w^{\prime}\right) \subset C(w),
$$

where the union is over all $w^{\prime \prime}<w^{\prime}$ with $l\left(s_{i} \cdot w^{\prime \prime}\right)<l\left(w^{\prime \prime}\right)$. Then our map is a stratawise isomorphism (thus a universal homeomorphism) over $C(w)-Z$. Over a point in $z \in Z$ the fibre is isomorphic to $\mathbb{P}^{1}$.

This already implies geometric connectedness, which in turn trivially implies b). For c) then note that the maps $\tilde{C}\left(w^{\prime \prime}\right) \rightarrow \tilde{C}(w)$ are universally injective. As $\tilde{C}\left(w^{\prime}\right)$ and its image are compatibly Frobenius-split they must be closed immersions (the extension of function-fields is purely inseparable, thus trivial because of the splitting).

Finally for the direct images of $\pi_{w}$ we now factor it as

$$
D(w)=\mathcal{P}_{i} \times{ }^{\mathcal{B}} D\left(w^{\prime}\right) \rightarrow \mathcal{P}_{i} \times{ }^{\mathcal{B}} \tilde{C}\left(w^{\prime}\right) \rightarrow \tilde{C}(w),
$$

and denote by $\tilde{Z} \subset \tilde{C}\left(w^{\prime}\right)$ the reduced subscheme which is the union of all $\tilde{C}\left(w^{\prime \prime}\right)$, for $w^{\prime \prime}<w^{\prime}$ and $l\left(s_{i} \cdot w^{\prime \prime}\right)=l\left(w^{\prime \prime}\right)-1$. Then the map is isomorphic over the complement of $\tilde{Z}$, while for any $z \in \tilde{Z}$ the fiber is isomorphic to $\mathbb{P}^{1}$, as a scheme. To check that the higher direct images are trivial in such a $z$ it suffices by the comparison theorem for proper maps that the formal completion of $\mathcal{P}_{i} \times{ }^{\mathcal{B}} \tilde{C}\left(w^{\prime}\right)$ along the fibre $\mathbb{P}^{1}$ has trivial cohomology. However this follows easily from the fact that globally generated sheaves on $\mathbb{P}^{1}$ have trivial higher cohomology.

As remarked in [11, Th. 5] the proof of Ramanathan in [13] carries over, so also all higher $\mathbb{R} \pi_{w, *}^{i}\left(\omega_{D(w)}\right)$ vanish, and $\tilde{C}(w)$ is Cohen-Macaulay. Also we obtain flat 
models for the $\tilde{C}(w)$ over the integers $\mathbb{Z}$ : Namely obviously the $D(w)$ are naturally defined as smooth projective schemes over $\mathbb{Z}$. Although we do yet not know that $\mathcal{X}_{G}$ is flat over $\mathbb{Z}$, nevertheless we may define $\tilde{C}(w)$ as the affine scheme over it whose algebra is $\pi_{w, *}\left(\mathcal{O}_{D(w)}\right)$. The vanishing of higher direct images implies that this is flat over $\mathbb{Z}$ and commutes with base-change. Also for $w^{\prime} \leq w \tilde{C}\left(w^{\prime}\right)$ is a closed subscheme of $\tilde{C}(w)$, and the $\tilde{C}(w)$ 's (indexed by $W^{\text {aff }}$ with its Bruhat-order) form an ind-scheme $\tilde{C}_{G}$ mapping to $\mathcal{X}_{G}$. Also for each simple affine roots $\alpha_{i}$ there is a cofinal set of $w$ 's with $C(w)$ stable under the corresponding $S L_{2}$, that is with $l\left(s_{i} \cdot w\right)=l(w)-1$. It follows that the corresponding $S L_{2}$ also operates on $\tilde{C}_{G}$.

We shall finish the proof of the theorem by showing that it is a closed subscheme of $\mathcal{X}_{G}$. We already know that this holds in characteristic zero, by [8]. As his definition of Schubert-varieties is also a little bit complicated we repeat his basic argument here:

Choose a line-bundle $\mathcal{L}$ on $\mathcal{X}_{G}$ which is equivariant under a central extension of $L G$ and has positive degree on each line $\mathbb{P}^{1}$ corresponding to a simple affine root. Such an $\mathcal{L}$ can be obtained by pullback via an embedding $G \subseteq S L_{d}$. Form (over the base $\mathbb{Q}$ ) the inductive limits

$$
E(\mathcal{L})=\operatorname{Lim}_{\rightarrow} \Gamma(C(w), \mathcal{L})^{d u a l}
$$

and

$$
\tilde{E}(\mathcal{L})=\underset{\rightarrow}{\operatorname{Lim}} \Gamma(\tilde{C}(w), \mathcal{L})^{d u a l} .
$$

These are both integrable modules (over the Lie-algebra) with a highest weight vector of multiplicity one. Thus they are irreducible and coincide, so (by duality) $\mathcal{L}(\tilde{\lambda})$ has the same global section over $\mathcal{X}_{G}$ as over the inductive limit of all $\tilde{C}(w)$. As the transition maps are surjective for the latter it follows that also for each individual $w$ the global sections of $\mathcal{L}$ over $C(w)$ and $\tilde{C}(w)$ coincide. As $\mathcal{L}$ can be chosen such that is very ample on $C(w)$ the assertion follows.

Also for each $n$ the multiplication of sections defines a homomorphism

$$
E\left(\mathcal{L}^{\otimes n}\right) \rightarrow E(\mathcal{L})^{\otimes n},
$$

which is injective because of irreducibility. It follows that for each $w \Gamma(C(w), \mathcal{L})^{\otimes n}$ surjects onto $\Gamma\left(C(w), \mathcal{L}^{\otimes n}\right)$. As for big $n$ the latter space defines a projective embedding this holds true already for $\Gamma(C(w), \mathcal{L})$, and the global sections $\Gamma\left(\mathcal{X}_{G}, \mathcal{L}\right)$ (equal to the dual of $E(\mathcal{L})$ ) define a projective embedding of $\mathcal{X}_{G}$. Thus our $\mathcal{X}_{G}$ coincides with the one defined in [8] via such a projective embedding. This finishes our excursion into characteristic zero.

For the general case consider the formal completion $\hat{\mathcal{N}}^{-}$of $\mathcal{N}^{-}$. It is the indscheme whose $R$-valued points are elements of $G\left(R\left[t^{-1}\right]\right)$ which are $\equiv 1$ modulo a nilpotent ideal of $R$, and whose constant term lies in $N^{-}$. It is defined over the integers $\mathbb{Z}$. We claim that it is indrepresented by a power-series ring $\mathcal{R}=\mathbb{Z}\left[\left[u_{i}\right]\right]$ in infinitely many variables $\left\{u_{j} \mid i=1,2, \ldots\right\}$. That is if we call ideal $\mathcal{J} \subset \mathcal{R}$ open if it contains almost all of the $\left\{u_{j}\right\}$, and some power of the remaining ones, then $\mathcal{R}$ represents the ind-scheme

$$
\underset{\rightarrow}{\operatorname{Lim} \operatorname{Spec}}(\mathcal{R} / \mathcal{J})
$$


Lemma 10 a) $\hat{\mathcal{N}}^{-}$is indrepresented by a ring of formal power-series.

b) For any $g \in \hat{\mathcal{N}}^{-}(R)$ the image of $g$ in $L G(R)$ lies in the subgroup generated by the images of elements of $S L_{2}(R)$, under the maps $S L_{2} \rightarrow L G$ associated to the simple affine roots.

Proof Assertion a) follows easily from the fact that $\hat{\mathcal{N}}^{-}$is the product of the completions of $L^{--} T$ and of formal additive groups associated to negative affine roots. For b) the subgroup of $L G(R)$ generated by the images of $S L_{2}(R)$ contains the affine Weyl-group and thus all the additive groups $U_{\alpha}(R)$ corresponding to affine roots. Also $L^{--} T(R)$ is generated by the images of one-parameter groups $H_{\alpha}$, hence we can reduce to $G=S L_{2}$. There everything comes down to the famous all important formula ( $u$ a unit)

$$
\left(\begin{array}{cc}
u & 0 \\
0 & u^{-1}
\end{array}\right)=\left(\begin{array}{ll}
1 & u \\
0 & 1
\end{array}\right)\left(\begin{array}{cc}
1 & 0 \\
-u^{-1} & 1
\end{array}\right)\left(\begin{array}{ll}
1 & u \\
0 & 1
\end{array}\right)\left(\begin{array}{cc}
0 & -1 \\
1 & 0
\end{array}\right) .
$$

Now finally we can finish the proof of the theorem. Consider a torsion-free $\mathbb{Z}$-algebra $R$ and an element $g \in \hat{\mathcal{N}}^{-}(R)$, or equivalently its image $z=g * \in \mathcal{X}_{G}(R)$. As $g$ lies in the subgroup of $L G$ generated by $S L_{2}(R)$ 's and as these operate on $\tilde{C}_{G}, z$ lifts to a point $\tilde{z} \in \tilde{C}_{G}(R)$, that is to a $\tilde{z} \in \tilde{C}(\tilde{w})$. As $\tilde{C}(\tilde{w}) \rightarrow C(\tilde{w})$ is a universal homeomorphism this point also lies in the formal completion at the origin. Equivalently, if we denote by $\mathcal{A}$ the topological $\mathbb{Z}$-algebra which is the projective limit (with surjective transition maps) of the formal completions $A$ of the local rings of $\tilde{C}(w)$ at the origin, the map $\mathcal{R} \rightarrow R$ lifts to a continuous $\mathcal{A} \rightarrow R$. Furthermore this lift is unique, as $R$ is torsion-free and $\tilde{C}(w)_{\mathbb{Q}} \rightarrow C(w)_{\mathbb{Q}}$ is an isomorphism. Thus in the limit the natural map $\mathcal{R} \rightarrow \mathcal{A}$ admits a section $\mathcal{A} \rightarrow \mathcal{R}$.

We claim that the composition $\mathcal{A} \rightarrow \mathcal{R} \rightarrow \mathcal{A}$ is equal to the identity as well. For this denote by $A$ the ring of the completion of some $\tilde{C}(w)$. Lifting the origin to a $\mathbb{Z}$-valued point of $D(w)$ defines an injection $A \subseteq B$, with $B$ the ring of the formal completion at the lift which is a ring of formal powerseries over $\mathbb{Z}$. Now it suffices if the two endomorphisms of $\mathcal{A}$ become equal after composing $\mathcal{A} \rightarrow A \rightarrow B \rightarrow B_{n}$, where $B_{n}$ is the quotient of $B$ modulo some power of its defining ideal. However these two compositions correspond to $B_{n}$-valued points of $\tilde{C}(w)$ which become equal in $C(w)$. But then they are actually equal, as $B_{n}$ is torsion-free and the map $\tilde{C}(w)_{\mathbb{Q}} \rightarrow C(w)_{\mathbb{Q}}$ is an isomorphism.

We derive that $\mathcal{R} \cong \mathcal{A}$, and that the normalisation $\tilde{C}(w) \rightarrow C(w)$ is an isomorphism near the origin, because it induces a surjection on completed local rings.

Finally over any algebraically closed field $k$ the locus where $C(w)$ is not normal is Zariski-closed and stable under $\mathcal{B}^{+}$. If it is non-empty it must contain a $\mathcal{B}^{+}$fixedpoint, which must be $*$.

Remark. The result implies normality of Schubert-varieties also in incomplete flag-varieties, like $\mathcal{D}_{G}$. Namely their preimage in $\mathcal{X}_{G}$ is also a Schubert-variety.

Corollary 11 The closed immersion $\tilde{C}_{G} \subseteq \mathcal{X}_{G}$ is an isomorphism. 
Proof We must show that for any finitely generated $\mathbb{Z}$-algebra $R$ any element $x \in \mathcal{X}_{G}(R)$ lies in some $C(w)(R)$. Equivalently pullback by $x$ gives decreasing sequence of ideals $I_{w} \subset R$, and we must show that some $I_{w}=(0)$.

We show this for any noetherian ring $R$. The annihilators of the ideals $I_{w}$ form an increasing sequence which stabilises. If they stabilise to a proper ideal localise in a minimal prime over it. So we may assume that $R$ is local, and the $I_{w}$ are modules of finite length, for large $w$. Then they must also stabilise to a non-zero subideal of finite length. Dividing by a suitable power of the maximal ideal $\mathfrak{m}$ we then can assume that $R$ is artinian.

Now we use that $\tilde{C}_{G}$ is invariant under $S L_{2}$ 's. If $k=R / \mathfrak{m}$ denotes the residuefield the induced $x(k) \in \mathcal{X}_{G}(k)$ lies in the orbit of the origin $*$, under the group generated by $S L_{2}(k)$ 's. Elements of this group lift to $S L_{2}(R)$, so we may assume that $x(k)=*$. But then we have seen in the proof of the previous lemma that the group generated by $S L_{2}(R)$ 's transforms $x$ into the origin $*$.

Corollary 12 The Picard-group of rigidified line-bundles on $\mathcal{X}_{G}$ is (over any connected base) isomorphic to $\mathbb{Z}^{l+1}$, with the isomorphism defined by degrees on the lines $\mathbb{P}^{1}$ corresponding to simple affine roots. The Picard-group of $\mathcal{D}_{G}$ is $\mathbb{Z}$.

Proof The degree-map is surjective (by Theorem 7). If $\mathcal{L}$ has trivial degree on each $\mathbb{P}^{1}$ its pullback to each $D(w)$ is trivial, as $D(w)$ is a successive $\mathbb{P}^{1}$-fibration and elements of its Picard-group are determined by their degrees on fibres. It follows that $\mathcal{L}$ is trivial on each $C(w)$ and also on $\mathcal{X}_{G}$.

The assertion for $\mathcal{D}_{G}$ follows by pullback.

Another application is the construction of integrable representations. Namely consider an "ample" line-bundle $\mathcal{L}$ on $\mathcal{X}_{G}$ (the restriction to each $C(w)$ is ample), that is $\mathcal{L}$ is the tensor product of strictly positive powers of the fundamental $\mathcal{L}_{i}$. Then by Frobenius-splitting in characteristic $p$ for $w^{\prime} \leq w$ the restriction map $\Gamma(C(w), \mathcal{L}) \rightarrow \Gamma\left(C\left(w^{\prime}\right), \mathcal{L}\right)$ is surjective, that these modules are torsion-free, and that all higher cohomology vanishes. We then form the module (with discrete topology)

$$
E(\mathcal{L})=\underset{\rightarrow}{\operatorname{Lim}} \Gamma(C(w), \mathcal{L})^{d u a l} .
$$

It is an inductive limit of finite free $\mathbb{Z}$-modules, with split injective transition maps, and a suitable central extension $\tilde{L} G$ of $L G$ will act on it. Also it admits a canonical $\mathcal{B}^{+}$-eigenvector $e(\mathcal{L})$, the "highest weight" vector, given by evaluation at $*$. It generates $E(\mathcal{L})$ as module over the $\tilde{L} G$. More precisely each $\Gamma(C(w), \mathcal{L})^{\text {dual }}$ is generated over $\mathbb{Z}$ by $\mathcal{B}^{+} w \cdot e(\mathcal{L})$ (as $\mathcal{B}^{+} w \cdot *$ is dense in $\left.C(w)\right)$. The dual $\Gamma\left(\tilde{X}_{G}, \mathcal{L}(\tilde{\lambda})\right)$ is the projective limit of free $\mathbb{Z}$-modules, with surjective transition maps. It is naturally a topological module, and $E(\tilde{\lambda})$ is its continuous dual. Finally the $\vartheta$ 's define an $\mathcal{N}^{-}$-invariant element of the dual, which takes the value 1 on $e(\mathcal{L})$.

Some more details: The class of the central extension associated to $\mathcal{L}$ is linear in $\mathcal{L}$, and trivial if $\mathcal{L}$ is defined by a character of $\mathcal{B}^{+}$(induced from a character of $T$ ). It follows that there exists one central extension

$$
\mathbb{G}_{m} \rightarrow \tilde{L} G \rightarrow L G
$$


which operates on all $\mathcal{L}$ 's. The preimage of $T$ in it is a torus $\tilde{T}$ of dimension $l+1$, and the one-parameter group $H_{1,-\theta}+H_{\theta}$ defines an isomorphism between $\mathbb{G}_{m}$ and the kernel of $\tilde{T} \rightarrow T$. Thus $\tilde{T}$ is the product of one-parameter subgroups corresponding to simple affine roots, the $\mathcal{L}$ 's are parametrised by characters $\tilde{\lambda}: \tilde{T} \rightarrow \mathbb{G}_{m}$, and for each simple affine root $\alpha \tilde{\lambda}\left(H_{\alpha}\right)$ is the degree of $\mathcal{L}(\tilde{\lambda})$ on the associated $\mathbb{P}^{1} \subset \mathcal{X}_{G}$. For dominant $\tilde{\lambda}$ (that is $\tilde{\lambda}\left(H_{\alpha}\right) \geq 0$ for each affine simple $\alpha$ ) the cohomology $H^{i}\left(\mathcal{X}_{G}, \mathcal{L}(\tilde{\lambda})\right)$ vanishes in positive degrees, while in degree 0 we obtain the dual of the integrable module $E(\mathcal{L}(\tilde{\lambda}))=E(\tilde{\lambda}) . E(\tilde{\lambda})$ contains a canonical $\tilde{B}^{+}$-eigenvector $e(\tilde{\lambda})$ of $\tilde{T}$-weight $\tilde{\lambda}$, which generates it as $\tilde{L} G$-module. Furthermore $L G, \tilde{L} G, \mathcal{X}_{G}$ and all $\mathcal{L}(\tilde{\lambda})$ admit a $\mathbb{G}_{m}$-action. A unit $u$ acts by sending $t$ to $u \cdot t$. This action is expanding on $L^{-} G$, so it follows that the global sections $\Gamma\left(\mathcal{X}_{G}, \mathcal{L}(\tilde{\lambda})\right)$ have all weights $\geq 0$, und dually $E(\tilde{\lambda})$ has weights $\leq 0$ (the dual is the product of its $\mathbb{G}_{m}$-eigenspaces). Furthermore the elements of weight 0 are the global sections $\Gamma\left(G / B^{+}, \mathcal{L}(\lambda)\right)$, respectively their duals. Finally for affine roots $(n+\alpha)$ the $L_{0}$-weight is $n$. Thus it is natural to speak of extended affine weights $(n+\tilde{\lambda})$. These additional gradings should not be confused with those defined by the kernel $\mathbb{G}_{m}$ of $\tilde{L} G \rightarrow L G$ : These are on $E(\tilde{\lambda})$ always equal to $c$, and on its dual to $-c$.

We denote these weights $L_{0}$-weights, since our $\mathbb{G}_{m}$ will appear as a subgroup of the Virasoro-group. Each $L_{0}$-weight in $E(\tilde{\lambda})$ has finite multiplicity, that is the eigenspace is a finite free $\mathbb{Z}$-module. Dually $\Gamma\left(\mathcal{X}_{G}, \mathcal{L}(\tilde{\lambda})\right)$ is the product of their duals, and has the product topology. It is naturally a topological module which is the projective limit of discrete finitely generated free modules. Similarly after base-extension to an arbitrary ground-ring $R$. Note that the construction of $E(\tilde{\lambda})$ commutes with basechange, while for the dual we need to use the completed tensorproduct. It is precisely to avoid such topological difficulties that we prefer to use $E(\tilde{\lambda})$.

Finally we return to loop-groups.

Proposition $13 E(\tilde{\lambda})$ contains a maximal proper submodule, with irreducible quotient $F(\tilde{\lambda})$. The image $f(\tilde{\lambda})$ of e $(\tilde{\lambda})$ is the unique non-trivial $\mathcal{B}^{+}$-eigenvector in $F(\tilde{\lambda})$, and it generates it as $\mathcal{N}^{-}$-module.

Also the $\tilde{L} G$-submodule of $\Gamma\left(\mathcal{X}_{G}, \mathcal{L}(\tilde{\lambda})\right)$ generated by $\vartheta(\tilde{\lambda})$ is topologically irreducible, and already generated by the $\mathcal{B}^{+}$-transforms of $\vartheta(\tilde{\lambda})$. It is the dual of $F(\tilde{\lambda})$.

Proof $e(\tilde{\lambda})$ is the only $\tilde{T}$-eigenvector of conformal weight 0 in $E(\tilde{\lambda})$. Thus ony $\tilde{L} G$-submodule of not containing it is annihilated by $\vartheta(\tilde{\lambda})$. Hence the perpendicular of the closed subspace generated by $\vartheta(\tilde{\lambda})$ is the maximal submodule not containing $e(\tilde{\lambda})$. In the quotient $F(\tilde{\lambda})$ any non-trivial submodule contains $f(\tilde{\lambda})$, and thus must coincide with $F(\tilde{\lambda})$ as $f(\tilde{\lambda})$ is a generator.

Now the $\tilde{L} G$-submodule generated by another $\mathcal{B}^{+}$-eigenvector $f \neq 0$ coincides with the $\mathcal{N}^{-}$-submodule generated by it: Namely for any linear form $v$ in the dual the function $v(g(f))$ defines an element of $\Gamma\left(\mathcal{X}_{G}, \mathcal{L}(\tilde{\lambda})\right)(\tilde{\lambda}$ the associated character). This vanishes identically if it vanishes on the dense orbit $\mathcal{N}^{-} \cdot *$. By irreducibility this must be all of $F(\tilde{\lambda})$, and by considering weights it follows that $f$ must coincide with $f(\tilde{\lambda})$. 
Finally the dual of $F(\tilde{\lambda})$ is topologically irreducible, thus generated by $\vartheta(\tilde{\lambda})$. Also since this element is $\mathcal{N}^{-}$-invariant the dual is already topologically generated by its $\mathcal{B}^{+}$-transforms, by a similar reasoning to the above.

\section{Virasoro-action}

We define the Virasoro-group $\mathcal{V}$ ir as a central extension of the ind-groupscheme $\mathcal{A} u t$ of automorphisms of the punctured formel disk. For each $R \mathcal{A} u t(R)$ is the set of continuous $R$-automorphisms of $R((t))$. Such an automorphism $\phi$ is determined by $\phi(t)$, which must be a Laurent-series

$$
\phi(t)=\sum a_{n} \cdot t^{n}
$$

with $a_{1}$ invertible, $a_{n}$ nilpotent for $n \leq 0$, and almost all $a_{n}$ with negative index $n$ vanish. It is obvious that this is representable by an affine ind-scheme.

The subgroup $\mathcal{A} u t^{+} \subset \mathcal{A} u t$ is the subgroup of automorphisms of $R[[t]]$ respecting the ideal $(t)$. It is the subscheme defined by $a_{n}=0$ for $n \leq 0$, and is an honest scheme, the product of $\mathbb{G}_{m}$ and an infinite number of copies of $\mathbb{A}^{1}$. It contains as subgroup the Borel $B_{V} \subset P G L_{2}$ of automorphisms of $\mathbb{P}^{1}$ respecting $\{\infty\}$ (Recall that $t$ is a local coordinate at $\infty$ ). Although we make no use of it this is the place to mention the subgroup $\widehat{P G L_{2}} \subset \mathcal{A} u t$ (an ind-scheme), the formal completion of $P G L_{2}$ along $B_{V}$.

Another important subgroup $\mathcal{A} u t^{-} \subset \mathcal{A} u t$ is the group of automorphisms of the affine line $\mathbb{P}^{1}-\{\infty\}$ respecting $\{0\}$. Such an automorphism $\phi$ sends $t^{-1}$ to a finite sum

$$
\phi\left(t^{-1}\right)=\sum_{n \geq 1} b_{n} \cdot t^{-n},
$$

with $b_{1}$ invertible, $b_{n}$ nilpotent for $n \geq 2$, and almost all of them $=0$. It is the semidirect product of $\mathbb{G}_{m}$ (giving $L_{0}$-weights) and the normal subgroup defined by $b_{1}=1$. The latter is a formal group, ind-represented by a powerseriesring in infinitely many variables. In terms of the coordinates $a_{n}$ it is defined by $a_{1}=1, a_{n}=0$ for $n \geq 2$. Finally

$$
\mathcal{A} u t=\mathcal{A} u t^{-} \cdot \mathcal{A} u t^{+},
$$

and

$$
\mathcal{A u t}^{-} \cap \mathcal{A} u t^{+}=\mathbb{G}_{m} .
$$

Obviously $\mathcal{A} u t$ acts (by change of variables) on $L G$, fixing $G$. Also $\mathcal{A} u t^{ \pm}$ respects $\mathcal{B}^{ \pm}$. The main result of this section is that this action extends to an action on $\tilde{L} G$, and to a projective action on each irreducible integrable module $F(\tilde{\lambda})$. The corresponding central extension of $\mathcal{A} u t$ will be called $\mathcal{V}$ ir (Virasoro-group).

Theorem 14 a) $\mathcal{A u t}^{+}$acts on $E(\tilde{\lambda})$ such that the action fixes the highest weightvector $e(\tilde{\lambda})$, and intertwines the original with the conjugated $\mathcal{B}^{+}$-action.

b) $\mathcal{A u t}^{-}$acts on the closed submodule of $\Gamma\left(\mathcal{X}_{G}, \mathcal{L}(\tilde{\lambda})\right)$ generated by the thetafunction $\theta(\tilde{\lambda})$, and intertwines the original with the conjugated $\mathcal{B}^{-}$-action. 
c) These two actions coincide on $\mathbb{G}_{m}$. Furthermore, over an algebraically closed field $k$, they they induce a projective action of $\mathcal{A}$ ut on $F(\tilde{\lambda})$ which intertwines the action of $\tilde{L} G$ with a conjugate action.

Proof We already know the action of the group $\mathbb{G}_{m}$ fixing $e(\tilde{\lambda})$ and $\vartheta(\tilde{\lambda})$.

For the rest of the proof we use that all these facts are already known in characteristic 0, thanks to the Suguwara-construction ([7, Ex. 18.8], [16, Lemma 1.2.2.]): This gives in characteristic 0 an action of the Lie-algebra of a central extension of $\mathcal{A} u t$ on the integrable modules and thus on $\mathcal{X}_{G}$. However apart from the $L_{0}$-factor $\mathbb{G}_{m} \mathcal{A} u t^{ \pm}$is prounipotent, respectively a formal group, and the actions integrate (for $\mathcal{A u t}^{+}$one also needs that $L_{0}$-weights are bounded above). Note however that we have normalised in such a way that $e(\tilde{\lambda})$ becomes $L_{0^{-}}$ invariant, which does not hold for the Sugawara-formula. The two actions differ by chosing different lifts of $\mathcal{A} u t^{ \pm}$into the central extension $\mathcal{V}$ ir.

Also $\mathcal{A} u t^{ \pm}$is flat over $\mathbb{Z}$, that is is an inductive limit of affines $\operatorname{Spec}(R)$ with $R$ torsion-free. It then suffices to construct functorial actions of $\mathcal{A} u t^{ \pm}(R)$ on $E(\tilde{\lambda}) \otimes R$, respectively the closed subspace of sections generated by $\vartheta(\tilde{\lambda})$, for such torsion-free rings $R$.

To prove a) use that ( $R$ torsion-free) the action of $\mathcal{A} u t^{+}(R)$ on $E(\tilde{\lambda}) \otimes R_{\mathbb{Q}}$ respects $E(\tilde{\lambda}) \otimes R$, because it is generated by $\tilde{L} G \cdot e(\tilde{\lambda})$.

For b) use the "dual" argument with $\vartheta(\tilde{\lambda})$.

Finally for c) we may assume that $k$ has characteristic $p>0$. Then the action of $\mathcal{A} u t^{+}$on $E(\tilde{\lambda})$ stabilises $e(\tilde{\lambda})$ and must respect the unique irreducible quotient $F(\tilde{\lambda})$. Its dual is the unique irreducible quotient of the subspace of $\Gamma\left(\mathcal{X}_{G}, \mathcal{L}(\tilde{\lambda})\right)$ generated by $\vartheta(\tilde{\lambda})$, and is respected by $\mathcal{A} u t^{-}$. As both actions coincide on the intersection we obtain a map from $\mathcal{A} u t=\mathcal{A} u t^{+} \cdot \mathcal{A} u t^{-}$into the automorphisms of $F(\tilde{\lambda})$, which intertwines the $\tilde{L} G$-action with its conjugate. By irreducibility this defines a projective representation of $\mathcal{A} u t$, or a representation of a central extension (which splits as a map of schemes) Vir $\rightarrow \mathcal{A} u t$.

Remarks. a) One can construct $\mathcal{V}$ ir-stable lattices in $E(\tilde{\lambda})_{\mathbb{Q}}$, for example the $\mathcal{A} u t^{-}$-span of $E(\tilde{\lambda})$.

b) The $\mathcal{A} u t^{+}$-action can also be defined via its geometric action on $\mathcal{X}_{G}$. It follows that it respects the comultiplication-map

$$
E(\tilde{\lambda}+\tilde{\mu}) \rightarrow E(\tilde{\lambda}) \otimes E(\tilde{\mu}),
$$

dual to multiplication on global sections. Also for weights $\tilde{\lambda}$ with $\lambda=0$ (that is parametrised by the central charge $c$ ) the geometric action of $\widehat{P G L} \widehat{D}_{2}$ on $\mathcal{D}_{G}$ defines an action on $E(\tilde{\lambda})$.

\section{Moduli-stacks of $G$-bundles}

For the beginning $G$ can be again an arbitrary reductive connected group-scheme, but at the end we shall restrict to simple, semisimple, and simply connected $G$ 's. Suppose $C \rightarrow S$ is a smooth projective curve, with geometrically irreducible fibres 
of constant genus $g$. The moduli-stack $\mathcal{M}_{G}$ classifies principal $G$-bundles $P$ on base-changes $C \times{ }_{S} S^{\prime}$.

It is shown in [5] (Th. 1) that one can find a Borel $\mathcal{B} \subset G_{P}$. Thus the structuregroup reduces to $B \subset G$, and we call $\mathcal{B}$ "generic" if for each simple positive root $\alpha$ the associated line-bundle $\mathcal{L}(-\alpha)$ is generated by its global sections and has trivial first cohomology. For example this holds if $\operatorname{deg}(\mathcal{L}(\alpha)) \leq-2 g$. That there exists a "generic" Borel follows also from [5].

Remark. If $G_{P}$ contains a Borel after basechange $S^{\prime} \rightarrow S$, it defines a classifying map

$$
S^{\prime} \times{ }_{S} C \rightarrow(G / B)_{P} .
$$

This map is smooth if $S^{\prime}$ is the classifying space of "generic" Borel's, by infinitesimal calculations as in [5] $\left(\mathcal{L i e}\left(G_{P}\right) / \mathcal{L} i e(\mathcal{B})\right.$ is globally generated).

Now assume that $C \rightarrow S$ has a section. We denote its image by $\{\infty\} \subset C$, and let $C^{0}=C-\{\infty\}$ denote the complement.

Proposition 15 Suppose $G$ is semisimple. For any $G$-torsor $P$ on $C$, there exists a flat finitely presented covering $S^{\prime} \rightarrow S$ such that the pullback of $P$ to $C^{0} \times{ }_{S} S^{\prime}$ is trivial. Moreover if $G$ is simply connected we may use an étale covering.

Proof See [5] (Th. 3).

As an application note that the sections of $G$ over $C^{0} \times{ }_{S} S^{\prime}$ are representable by an ind-group-scheme $R_{C^{0} / S}(G)$ (Weil-restriction). That one obtains an indgroupscheme is an easy generalisation of the discussion after Definition $1 . R_{C^{0} / S}(G)$ is the inductive limit of its closed subschemes where one restricts the pole-order at infinity of an element of $G$ and of its inverse.

Also suppose we have chosen an isomorphism between the formal completion of $C$ along $\{\infty\}$ and $\operatorname{Spf}\left(\mathcal{O}_{S}[[t]]\right)$. This is possible locally in the Zariski-topology of $S$. Recall that $\mathcal{M}_{G}$ denotes the algebraic ind-stack of principal $G$-torsors on $C$ (in either flat, smooth or étale topology).

Corollary 16 Suppose $G$ is semisimple. Then $\mathcal{M}_{G}=R_{C^{0} / S}(G) \backslash \mathcal{D}_{G}$. More precisely $\mathcal{D}_{G}$ is an $R_{C^{0} / S}(G)$-torsor over $\mathcal{M}_{G}$, in the flat topology (étale if $G$ is simply connected).

Proof We show that $\mathcal{D}_{G}$ represents the functor " $G$-bundles trivialised on $C$ ")"

Firstly if we have a trivialised $G$-bundle $P$ we can (flat locally) trivialise its restriction to $\{\infty\}$ and also $\operatorname{Spf}\left(\mathcal{O}_{S}[[t]]\right)$. Then we obtain by projective limits also a trivialisation on the pullback to $\operatorname{Spec}\left(\mathcal{O}_{S}[[t]]\right)$, and on $\operatorname{Spec}\left(\mathcal{O}_{S}((t))\right)$ the two trivialisations differ by an element of $g \in L G\left(\mathcal{O}_{S}\right)$, so that $g \cdot * \in \mathcal{D}_{G}\left(\mathcal{O}_{S}\right)$ is welldefined.

Conversely any point in $\mathcal{D}_{G}=L G / L^{+} G$ lifts Zariski-locally to $g \in L G$. This $g$ defines a $G$-torsor on $C$, by gluing the trivial torsors on $C^{0}$ and $\operatorname{Spec}\left(\mathcal{O}_{S}[[t]]\right)$. For example for $G$-module $E$ (over $\mathbb{Z}$ ) we obtain an associated sheaf $E_{G}$ such that $E_{G} \mid C^{0}=E \otimes \mathcal{O}$, and for an affine neighbourhood $U$ of $\{\infty\}$ a section $e \in \Gamma\left(U \cap C^{0}, E_{G}\right)$ extends to all of $U$ iff $g^{-1} \cdot e \in E[[t]]$. (That this gluing 
works is easily seen for noetherian rings, which suffices for us since everything can be defined over finitely generated $\mathbb{Z}$-algebras, and $\mathcal{D}_{G}$ is the inductive limit of schemes of finite type over $\mathbb{Z}$. For non-Noetherian bases this is still true, but requires more thought. See [1], [2] for details)

For $G=G L_{d}$ this suffices to construct a torsor. In general we have defined a tensor-functor from finitely generated torsion-free $\mathbb{Z}-G$-modules to vectorbundles on $C$, and extends by inductive limit to all torsion-free $G$-modules. Especially for a closed embedding $G \subseteq H=G L_{d}$ the ideal defining $G$ in $\mathcal{O}_{H}$ is stable under $G$ acting by left translations. Thus in the $H$-torsor $P_{H}$ (with right $H$-action) it defines a closed subscheme $P_{G} \subseteq P_{H}$, stable under $G$-multiplication from the right. One checks that it is faithfully flat and a $G$-torsor, and that it defines the tensorfunctor. Thus our inverse map.

\section{Linebundles on $\mathcal{M}_{G}$}

In this section $S$ denotes a noetherian base-scheme, $C \rightarrow S$ a smooth projective geometrically connected curve, with a section $\{\infty\}$, and $G$ a semisimple simply connected split groupscheme. We want to compute the group of linebundles $\mathcal{L}$ on $\mathcal{M}_{G}$ which are rigidified, that is trivialised over the origin (which corresponds to the trivial torsor). By the previous this is the same as $R_{C^{0} / S}(G)$-equivariant linebundles on $\mathcal{D}_{G}$, trivialised at the origin. These have as obvious invariant their central charge, that is the degree on the projective line $\mathbb{P}^{1} \subset \mathcal{D}_{G}$ corresponding to the affine root $\alpha_{0}=1-\theta$. We claim that the central charge classifies linebundles:

Theorem 17 Suppose $S$ is a connected noetherian base-scheme. Then the central charge defines an isomorphism from the group of rigidified linebundles on $\mathcal{M}_{G}$ to $\mathbb{Z}$.

Remarks. a) In characteristic zero this has been shown by C. Sorger ([14]), following [10], [3]. Because of uniqueness his line-bundles must coincide with ours. Alternatively once the line-bundles are known to exist the arguments of [6] prove (in characteristic zero) the Verlinde-formula for them, so their global sections coincide with the spaces of conformal blocks. As Sorger's construction starts from these he gets the same bundles as we do.

b) It would be nice to remove the hypotheses "noetherian".

c) For any representation $E$ of $G$ one obtains an $\mathcal{L}$ as determinant of cohomology. Its central charge can be computed as $c=\operatorname{tr}_{E}\left(H_{\theta}^{2}\right) / 2$. Also if $E$ is orthogonal it has a square-root, the "Pfaffian". These suffice to construct all central charges for classical groups and for $G_{2}$. However for $E_{8}$ there remains a factor 30 .

Proof We first construct a linebundle with central charge 1. Denote by $\left\{\alpha_{1}, \ldots, \alpha_{l}\right\}$ the simple roots of $G$ (semisimple, simple, and simply connected). We choose an invariant product on the cocharacters of the maximal torus $T$ such that

$$
<H_{\alpha_{i}}, H_{\alpha_{i}}>=2 \cdot a_{i},
$$

with $a_{i}=1$ for long roots $\alpha_{i}$, and $a_{i}=2$ or $a_{i}=3$ for short roots. Also we define a symmetric matrix $a_{i j}=<H_{\alpha_{i}}, H_{\alpha_{j}}>$. If $w_{0} \in W$ denotes the longest 
element in the Weyl-group, we define an involution on simple roots by the rule $\alpha_{i^{*}}=-w_{0}\left(\alpha_{i}\right)$.

Next we need some geometry on the space $X=G / B, B=T \cdot N^{+}$the Borel formed with the positive roots. On $X$ we have canonical $G$-equivariant line-bundles $\mathcal{L}(\lambda)$ corresponding to weights $\lambda: T \rightarrow \mathbb{G}_{m}$. The space of global sections $\Gamma(X, \mathcal{L}(\lambda))$ is identified with the space of regular functions $f$ on $G$ satisfying $f(g \cdot b)=\lambda(b) \cdot f(g)$. Evaluation at the origin defines a linear form $l_{\lambda}$ on $\Gamma(X, \mathcal{L}(\lambda)$, which is a $B$-eigenvector (in the dual) of weight $\lambda$. Also it contains a highest weight-vector $v_{\lambda}$ of weight $-w_{0}(\lambda)$, normalised so that $l_{\lambda}\left(w_{0}\left(v_{\lambda}\right)\right)=1$. Denote its zero-divisor by $V\left(v_{\lambda}\right)$. Especially important are the fundamental weights $\epsilon_{i}$ with $\epsilon_{i}\left(H_{\alpha_{j}}\right)=\delta_{i j}$.

Next consider the product $X \times X$, with the diagonal action of $G$. The $G$-orbits in it are indexed by the Weyl-group. The function

$$
d_{i}\left(g_{1}, g_{2}\right)=l_{\epsilon_{i}}\left(g_{2}^{-1} g_{1}\left(v_{\epsilon_{i}}\right)\right)
$$

defines a $G$-invariant global section

$$
d_{i} \in \Gamma\left(X \times X, \mathcal{L}\left(\epsilon_{i^{*}}, \epsilon_{i}\right)\right) .
$$

We denote its divisor by $D_{i} \subset X \times X$. It is the closure of the $G$-orbit through $\left(w_{0}, s_{i}\right)$. Furthermore if $W_{\neq i} \subset W$ denotes the subgroup generated by reflections $s_{j}$ with $j \neq i, d_{i}$ takes the value 1 on $\left(w_{0}, W_{\neq i}\right)$.

Next we define a $G$-invariant section

$$
e_{i} \in \Gamma\left(D_{i}, \mathcal{L}\left(\epsilon_{i^{*}}, \epsilon_{i}-\alpha_{i}\right)\right)
$$

by the function

$$
e_{i}\left(g_{1}, g_{2}\right)=l_{\epsilon_{i}}\left(s_{i} g_{2}^{-1} g_{1}\left(v_{\epsilon_{i}}\right)\right) .
$$

(one checks without much difficulty that this is well-defined on $D_{i}$ ). It is normalised by the rule $e_{i}\left(w_{0}, W_{\neq i} \cdot s_{i}\right)=1$. Note that

$$
\epsilon_{i}-\alpha_{i}=-\epsilon_{i}-\sum_{j \neq i} \alpha_{i}\left(H_{\alpha_{j}}\right) \cdot \epsilon_{j}
$$

Similarly we can define

$$
\tilde{e}_{i} \in \Gamma\left(D_{i}, \mathcal{L}\left(\epsilon_{i^{*}}-\alpha_{i^{*}}, \epsilon_{i}\right)\right)
$$

by

$$
\tilde{e}_{i}\left(g_{1}, g_{2}\right)=l_{\epsilon_{i}}\left(g_{2}^{-1} g_{1} s_{i}\left(v_{\epsilon_{i}}\right)\right),
$$

and $\tilde{e}_{i}\left(w_{0}, s_{i} \cdot W_{\neq i}\right)=1$. Finally one has the following equality (both sides are $G$-invariant and coincide on $\left.\left(w_{0}, s_{i}\right)\right)$ :

$$
\left(e_{i} \cdot \tilde{e}_{i}\right)^{a_{i}}=\prod_{j \neq i}\left(s_{j} \mid D_{i}\right)^{-a_{i j}},
$$

as section of

$$
\bigotimes_{i \neq j} \mathcal{L}\left(\epsilon_{j^{*}}, \epsilon_{j}\right)^{-a_{i j}}
$$


The divisor of $e_{i}$ is contained in the union of the closures of the $G$-orbits through $\left(w_{0}, s_{i} s_{j}\right)$, where $j \neq i$ and $s_{i}$ and $s_{j}$ do not commute (These are the only possible codimension-2 orbits in $D_{i}$ not excluded by the previous). Similarly for $\tilde{e}_{i}$ we get the orbits through $\left(w_{0}, s_{j} s_{i}\right)$. From the equation above one sees that the multiplicities of these orbits are equal to $-a_{i j} / a_{i}$ times the multiplicities in $D_{i} \cap D_{j}$.

Finally we shall need a $T$-invariant deformation. Consider the strictly dominant one-parameter group $\rho: \mathbb{G}_{m} \rightarrow T$ with $\alpha_{i}(\rho)=1,1 \leq i \leq l$. It acts on $X \times X$ via $(\rho(t), 1)$. We show that the limits $\lim _{t \rightarrow 0, \infty}$ of our objects exist, that is we obtain sections of line-bundles on $(X \times X) \times \mathbb{P}^{1}$. We only consider $t \rightarrow \infty$, the other limit being similar. Also note that the conjugate $(\rho(t), 1)(G) \subset G \times G$ extends to a flat subgroup, whose fibre at $\{\infty\}$ is the product of the diagonal $T$ with $N^{+} \times N^{-}$. The limits of our sections will be invariant under this group, which will determine them up to a constant factor.

Lemma $18(\rho(t), 1)\left(d_{i}\right)$ extends to a global section of $\mathcal{L}\left(\epsilon_{i^{*}}, \epsilon_{i}\right) \otimes \mathcal{O}\left(2 \epsilon_{i}(\rho)\right)$ on $X \times X \times \mathbb{P}^{1}$. Its divisor $\mathcal{D}_{i}$ is flat over $\mathbb{P}^{1}$, and regular outside a closed subset of codimension $\geq 2$ in each fibre. Its intersection with $X \times X \times\{\infty\}$ has two irreducible components, namely $V\left(v_{\epsilon_{i^{*}}}\right) \times X$ and $X \times w_{0}\left(V\left(v_{\epsilon_{i}}\right)\right)$. Similarly the intersection with $X \times X \times\{0\}$ has two components $w_{0}\left(V\left(v_{\epsilon_{i^{*}}}\right)\right) \times X$ and $X \times V\left(v_{\epsilon_{i}}\right)$.

Proof In the limit

$$
\lim _{t \rightarrow \infty} t^{-\epsilon_{i^{*}}(\rho)}(\rho(t), 1)\left(d_{i}\right)=v_{\epsilon_{i^{*}}} \otimes w_{0}\left(v_{\epsilon_{i}}\right):
$$

Namely firstly the limit exists and has first component $v_{\epsilon_{i^{*}}}$. Next it is $N^{+} \times N^{-}$invariant, and takes value 1 on $\left(w_{0}, 1\right)$. This (and the analogous assertion at $\{0\}$ ) implies everything except regularity in relative codimension $\leq 1$.

That could only fail over $\{0\}$ or $\{\infty\}$. Over $\{\infty\}$ this can only happen if $\mathcal{D}_{i}$ is not regular along the generic point of $V\left(v_{\epsilon_{i}}\right) \times w_{0}\left(V\left(v_{\epsilon_{i}}\right)\right)$, the intersection of the two irreducible components. One point in this intersection is $\left(w_{0}, s_{i}\right)$. But

$$
t^{-\epsilon_{i^{*}}(\rho)}(\rho(t), 1)\left(d_{i}\right)\left(w_{0}, s_{i}\right)=t^{-\epsilon_{i^{*}}(\rho)} l_{\epsilon_{i}}\left(s_{i} w_{0} \rho(t) s_{i}\left(v_{\epsilon_{i}}\right)\right)=t^{-\alpha_{i}(\rho)}=t^{-1},
$$

so the equation for $\mathcal{D}_{i}$ has order one on the constant section $\left(w_{0}, s_{i}\right)$. This ends the proof of the lemma.

Similarly the limit

$$
\lim _{t \rightarrow \infty} t^{-\epsilon_{i^{*}}(\rho)}(\rho(t), 1)\left(e_{i}\right)
$$

exists, vanishes on the first component and is equal to $\left(v_{\epsilon_{i *}^{*}} \nabla s_{i} w_{0}\left(v_{\epsilon_{i}}\right)\right.$ on the second. However if we remove this component from the divisor both restrictions become non-zero.

Note that after this operation we obtain a reflexive sheaf on $\mathcal{D}_{i}$, which will be locally free outside a closed subset of relative codimension $\geq 2$. Especially it makes sense to restrict to the fibre at $\{\infty\}$. Also the restrictions of the linebundle (better:reflexive sheaf) to the components of the fibre will change by the normal-bundle, so we obtain $\mathcal{L}\left(\epsilon_{i^{*}}\right) \otimes \prod_{j \neq i} \mathcal{L}\left(\epsilon_{j}\right)^{-\alpha_{i}\left(H_{\alpha_{j}}\right)}$, and $\mathcal{O} \otimes \mathcal{L}_{i}$. 
Lemma $19 t^{-\epsilon_{i^{*}(\rho)}}(\rho(t), 1)\left(e_{i}\right)$ extends to a global section of

$\left.\left.\mathcal{L}\left(\epsilon_{i^{*}}, \epsilon_{i}-\alpha_{i}\right) \otimes \mathcal{O}\left(2 \epsilon_{i}(\rho)\right)\left(-\left(w_{0}\left(V\left(v_{\epsilon^{*}}\right)\right) \times X \times\{0\}\right)\right)-\left(V\left(v_{\epsilon^{*}}\right) \times X \times\{\infty\}\right)\right)\right)$

on $\mathcal{D}_{i}$. At $\{\infty\}$ its restriction to the two components are non-zero, and proportional to the products of the unique $N^{ \pm}$-invariant sections.

Proof We only have to show that the restrictions do not vanish. For this compute the value on $\left(w_{0}, s_{i}\right)$ (which we did already in the previous proof).

This lemma will be applied to the product of four copies of $X$. We consider the pullback of the situation of the previous lemma (divisor with section of a linebundle over it) once via the coordinates $(1,3)$ and $(2,4)$, and once via $(1,4)$ and $(2,3)$. After the degeneration $(t \rightarrow \infty)$ we shall obtain in totality the same (four) irreducible components, and the same sections of line-bundles over them.

Now finally we can construct our line-bundle $\mathcal{L}$ with central charge 1 . It suffices to do this for curves over a regular base-scheme $S$ (compatible with basechange), since the moduli-stack of curves is smooth. We have to associate to any $G$-torsor $P$ on $C \rightarrow S$ a line-bundle $\mathcal{L}(P)$ on $S$. We first do this if $G_{P}$ admits a "generic" Borel, and then use descent to show that the construction is independant of it.

We use the Borel as follows: The $G$-equivariant line-bundles $\mathcal{L}\left(\epsilon_{i}\right)$ on $X$ define line-bundles on the twist $X_{P}$ (a scheme over $C$ ). The Borel gives a section of $X_{P}$, and by pullback we obtain line-bundles $\mathcal{L}_{i}$ on $C$.

Next recall $([4,6.1])$ that from two line-bundles $\mathcal{A}$ and $\mathcal{B}$ on $C$ one can construct a line-bundle $\langle\mathcal{A}, \mathcal{B}\rangle$ on the base $S$ (assumed regular), as follows: Choose isomorphisms $\alpha: \mathcal{O}_{C}(A) \cong \mathcal{A}, \beta: \mathcal{O}_{C}(B) \cong \mathcal{B}$, for two coprime divisors $A$ and $B$ on $C$. Then we can form the intersection-cycle $A \cdot B$ and its pushforward $\pi_{*}(A \cdot B)$ on $S$. (For an irreducible cycle $Z \subset C$, its direct image $\pi_{*}(Z)$ vanishes if $Z$ is not generically finite over its image $\pi(Z)$. Otherwise $\pi_{*}(Z)=[k(Z): k(\pi(Z)] \cdot \pi(Z)$.) Then $\alpha$ and $\beta$ define an isomorphism

$$
<\alpha, \beta>: \mathcal{O}_{S}\left(\pi_{*}(A \cdot B)\right) \cong<\mathcal{A}, \mathcal{B}>.
$$

Multiplying $\alpha$ by a local function $f$ on $S$ multiplies $<\alpha, \beta>$ by $f^{\operatorname{deg}(\mathcal{B})}$, and similar for $\beta$. More generally an isomorphism between $\mathcal{O}_{C}(A)$ 's or $\mathcal{O}_{C}(B)$ 's is given by a rational function, and induces an isomorphism on the bracket. From this it follows that the bracket is well-defined. It is bilinear and symmetric in $\mathcal{A}$ and $\mathcal{B}$, with no difficulty about signs except that the symmetry on $\langle\mathcal{A}, \mathcal{A}\rangle$ is equal to $(-1)^{\operatorname{deg}(\mathcal{A})}$. Also if $A$ is finite over $S,<\mathcal{O}(A), \mathcal{B}>$ is isomorphic to the norm of $\mathcal{B} \mid A$.

More generally one can define the meromorphic section $\langle\alpha, \beta>$ if $\beta$ is only a generator of $\mathcal{B}$ in the generic points of the irreducible components of the divisor $A$ of $\alpha$. Also the construction $<\mathcal{A}, \mathcal{B}>$ behaves well under base-change $S^{\prime} \rightarrow S$. The same holds for $\langle\alpha, \beta\rangle$ if the basechange is smooth.

Now, going back to torsors, we choose a covering $S^{\prime} \rightarrow S$ and a Borel in $G_{P} \times{ }_{S} S^{\prime}$ such that the associated map $C \times{ }_{S} S^{\prime} \rightarrow X_{P}$ is smooth. For example $S^{\prime}$ might classify "generic" Borel's. Then define the line-bundle $\mathcal{L}(P)$ on $S^{\prime}$ 
as

$$
\mathcal{L}(P)=-\sum_{i} a_{i}<\mathcal{L}_{i}, \mathcal{L}_{i}>-\sum_{i<j} a_{i j}<\mathcal{L}_{i}, \mathcal{L}_{j}>.
$$

(For simplicity we have switched to additive notation.)

If $S^{\prime \prime}=S^{\prime} \times{ }_{S} S^{\prime}$, we define an isomorphism

$$
\phi: p r_{1}^{*}(\mathcal{L}(P)) \cong p r_{2}^{*}(\mathcal{L}(P)),
$$

as follows:

As the divisors $D_{i} \subset X \times X$ as well as the sections $d_{i}, e_{i}$ are all $G$-invariant, they define divisors respectively sections of line-bundles on $(X \times X)_{P}$, and by smooth pullback on $C \times{ }_{S} S^{\prime \prime}$. Especially we pullback the linear combination (using again additive notation)

$$
\sum_{i} a_{i}<d_{i}, e_{i}>+\sum_{i<j} a_{i j}<d_{i}, d_{j}>.
$$

It defines a meromorphic section of

$$
\begin{aligned}
\sum_{i}<\mathcal{L}_{i^{*}}^{(1)}+\mathcal{L}_{i}^{(2)}, a_{i}\left(\mathcal{L}_{i^{*}}^{(1)}-\mathcal{L}_{i}^{(2)}\right)- & \sum_{j \neq i} a_{i j} \mathcal{L}_{j}^{(2)}> \\
& +\sum_{i<j} a_{i j}<\mathcal{L}_{i^{*}}^{(1)}+\mathcal{L}_{i}^{(2)}, \mathcal{L}_{j^{*}}^{(1)}+\mathcal{L}_{j}^{(2)}>.
\end{aligned}
$$

One notes that the "mixed terms" $<\mathcal{L}_{i^{*}}^{(1)}, \mathcal{L}_{j}^{(2)}>$ cancel because of symmetry (of $a_{i j}$ and $<,>$ ). What remains is

$$
\begin{aligned}
\sum_{i} a_{i}\left(<\mathcal{L}_{i}^{(1)}, \mathcal{L}_{i}^{(1)}>-<\right. & \left.\mathcal{L}_{i}^{(2)}, \mathcal{L}_{i}^{(2)}>\right) \\
& +\sum_{i<j} a_{i j}\left(<\mathcal{L}_{i}^{(1)}, \mathcal{L}_{j}^{(1)}>-<\mathcal{L}_{i}^{(2)}, \mathcal{L}_{j}^{(2)}>\right) \\
& =\operatorname{pr}_{2}^{*}(\mathcal{L}(P))-\operatorname{pr}_{1}^{*}(\mathcal{L}(P)) .
\end{aligned}
$$

Furthermore the divisor of this meromorphic section is the projection of the sum of the divisors of $a_{i} \cdot e_{i}$ (on $\left.D_{i}\right)$ and of $a_{i j}\left(D_{i} \cdot D_{j}\right)$. We claim that this sum vanishes:

This can be checked on $X \times X$. However then all these divisors are linear combinations of the closures of $G$-orbits through $\left(w_{0}, s_{i} s_{j}\right)$, for different (and noncommuting) reflections $s_{i}, s_{j}$. The multiplicities have been computed before, and they cancel indeed.

Hence the conclusion is that we have defined an isomorphism

$$
\phi: p r_{1}^{*}(\mathcal{L}(P)) \cong p r_{2}^{*}(\mathcal{L}(P)) .
$$

Before constructing a descent-datum out of $\phi$ we need some transitivity. For this consider the product $S^{(4)}$ of 4 copies of $S^{\prime}$, classifying a 4-tuple of generic Borel's. On it we have various isomorphisms

$$
\phi_{a b}: \operatorname{pr}_{a}^{*}(\mathcal{L}(P)) \cong p r_{b}^{*}(\mathcal{L}(P)) .
$$




\section{Lemma 20}

$$
\phi_{13} \cdot \phi_{24}=\phi_{14} \cdot \phi_{23}
$$

Proof It suffices to check after faithfully flat base-change, so we may assume that $G_{P}$ admits a Borel (not to be confused with the four Borel's classified by $S^{(4)}$ ). Furthermore over $S \times \mathbb{A}^{1}$ we can deform $P$ into a $T$-torsor. As the desired equality holds up to an invertible function (which is constant on the second factor $\mathbb{A}^{1}$ ) we may thus assume that $P$ is actually a $T$-torsor. Thus all $T$-invariant constructions on $X$ and its powers make sense on $X_{P}$, and we can use our previous deformationprocedure. Also it now suffices to check over a generic point of $S^{(4)}$, that is we may assume that $C$ and $P$ are defined over a field $k$, and our base is a field $K \supset k$. Furthermore the $k$-map $C \otimes_{k} K \rightarrow X_{P}^{4}$ is generic over $k$, that is dominant and its image avoids all subvarieties of codimension $\geq 2$ which are defined over $k$.

Next we perform our deformation-procedure on $X^{4} \times \mathbb{P}^{1}$, that is we transform over $\mathbb{G}_{m}$ with $(\rho(t), \rho(t), 1,1)$, and take the closure. Then $d_{i, a b}=p r_{a, b}^{*}\left(d_{i}\right)(a=$ $1,2, b=3,4)$ extend to sections of

$$
\mathcal{L}\left(\epsilon_{i^{*}}, \epsilon_{i}\right)^{(a, b)} \otimes \mathcal{O}\left(2 \epsilon_{i}(\rho)\right),
$$

with divisor $\mathcal{D}_{i, a b}$. Furthermore the section $e_{i, a b}$ gives a global section of the restriction to $\mathcal{D}_{i, a b}$ of

$$
\mathcal{L}\left(\epsilon_{i^{*}}, \epsilon_{i}+\alpha_{i}\right)^{(a, b)} \otimes \mathcal{O}\left(2 \epsilon_{i}(\rho)\right),
$$

which vanishes on $p_{b}^{-1}\left(w_{0}\left(V\left(v_{\epsilon_{i^{*}}}\right)\right) \times\{0\}\right.$ and $\operatorname{pr}_{b}^{-1}\left(\left(V\left(v_{\epsilon_{i^{*}}}\right) \times\{\infty\}\right.\right.$. These are Cartier-divisors on $\mathcal{D}_{1, a b}$ outside a $k$-defined closed subset of sufficiently high codimension, thus after pullback to $\left(C \times \mathbb{P}^{1}\right) \otimes_{k} K$ we can substract them, and the $e_{i, a b}$ become sections of a certain line-bundle on the pullback of $\mathcal{D}_{i, a b}$. This pullback will be finite and flat over $\mathbb{P}^{1} \otimes_{k} K$, and thus we may form the norm of these sections, which become global sections of a certain line-bundle on $\mathbb{P}^{1} \otimes_{k} K$. This is the basic idea, although its execution will turn out a little different. Namely we do not modify the line-bundles and work instead with meromorphic sections.

We repeat the same with $d_{j, a b} \mid \mathcal{D}_{i, a b}$, only that now there is no need to substract any divisors over $\{0\}$ or $\{\infty\}$. Finally we multiply these with $a_{i}$ respectively with $a_{i j}$, take the product of these sections once for the pairs $(a, b)=(1,3),(2,4)$, and once for $(a, b)=(1,4),(2,3)$, and then form the quotient $\phi_{13} \cdot \phi_{24} / \phi_{14} \cdot \phi_{23}$. It defines a regular section of the trivial line-bundle on $\mathbb{G}_{m} \otimes_{k} K$, that is a function. We claim that this function is regular with value 1 in $\{0, \infty\}$. This implies our claim (evaluate at $t=1$ ).

Let us check at $\{t=\infty\}$ : Over the formal completion of $\mathbb{P}^{1} \otimes_{k} K$ at $\{t=\infty\}$ the divisors $\mathcal{D}_{i, a b}$ are the disjoint sum of two disjoint divisors specialising to the two components over $\{t=\infty\}$. Modify the sections $e_{i, a b}$ by multiplying them by $t^{\epsilon_{i *}}$ on the the part specialising to $p r_{b}^{-1}\left(V\left(v_{\epsilon_{i *}}\right) \times X\right)$. We thus get new sections $\tilde{e}_{i, a b}$ which are regular and everywhere (on the formal completion) nonzero. Thus if we replace in the definition of $\phi_{a b}$ each $e_{i, a b}$ by $\tilde{e}_{i, a b}$, the result will be a regular nonzero section of the corresponding constant line-bundle. It differs from the original $\phi_{a b}$ 
by some power of $t$ which is easily computable from the degrees of the divisors $\operatorname{pr}_{b}^{-1}\left(w_{0}\left(V\left(v_{\epsilon_{i^{*}}}\right)\right)\right.$. It follows that these $t$-powers cancel in $\phi_{13} \cdot \phi_{24} / \phi_{14} \cdot \phi_{23}$. Hence this function is also regular at $\{t=\infty\}$.

To see that it takes the value 1 we use the modified $\tilde{e}_{i}$. Consider each summand $<d_{i}, \tilde{e}_{i}>$ respectively $<d_{i}, d_{j}>$ separately. In each case the restriction to $\{\infty\}$ of the divisors $\mathcal{D}_{i, a b}$ have two irreducible components which are pullbacks of the divisors $V\left(v_{\epsilon_{i}}\right)$ respectively $V\left(w_{0}\left(v_{\epsilon_{i}}\right)\right)$ on the factors. Thus we obtain the product (over $1 \leq a \leq 4$ ) of (the pullbacks of) the $N^{ \pm}$-invariant meromorphic section of a certain line-bundle (the same in both cases) on the $a$-th factor. These are only determined up to a factor, but these ambiguities cancel out.

Thus the quotient is 1 at $\{\infty\}$, and the lemma is shown.

Now going back to $S^{\prime \prime}=S^{\prime} \times{ }_{S} S^{\prime}$ the restriction of $\phi$ to the diagonal $S^{\prime}$ defines an automorphism of $\mathcal{L}(P)$. Multiply (say from the left) $\phi$ with its inverse to obtain a new isomorphism $\phi^{*}$ between $p r_{1}^{*}\left(\mathcal{L}(P)\right.$ and $p r_{1}^{*}\left(\mathcal{L}(P)\right.$. I claim that $\phi^{*}$ is a descent-datum:

We have to show (with hopefully obvious notation) that

$$
\phi_{13}^{*}=\phi_{12}^{*} \cdot \phi_{23}^{*},
$$

which means

$$
\phi_{13} \cdot \phi_{22}=\phi_{12} \cdot \phi_{23},
$$

and this follows from the lemma by setting " $2=4$ ", that is restricting to a partial diagonal.

So finally we obtain our $\mathcal{L}(P)$ on $S$. That it is independant from the choice of $S^{\prime}$ is clear (for two of them consider their disjoint union). Also we can rigidify it by substracting its value on the trivial torsor. It remains to show that $\mathcal{L}(P)$ has central charge 1 . It suffices to do this over a field $k$, so we can forget about rigidification.

To compute the central charge we form the $G$-torsor $P$ over $C \times \mathbb{P}^{1}$ obtained from the projective line $\mathbb{P}^{1} \subset \mathcal{D}_{G}$ corresponding to $\tilde{\alpha}_{0}=1-\theta$, and try to compute the degree of the line-bundle $\mathcal{L}(P)$ on $\mathbb{P}^{1}$. Evidently this depends only on the pullback of $\mathcal{L}(P)$ to the moduli-space of $S L_{2}$-torsors.

On this space we have a wellknown line-bundle of central charge 1: Namely an $S L_{2}$-torsor over $C \rightarrow S$ is a rank-2-bundle $\mathcal{E}$ with trivial determinant. Then $\operatorname{det}\left(H^{*}\left(C, \mathcal{O}^{2}\right)\right) \otimes \operatorname{det}\left(H^{*}(C, \mathcal{E})\right)^{\otimes-1}$ is the desired line-bundle on $S$. To see that its central-charge is indeed 1 one computes for the $S L_{2}$-torsor on $C \times \mathbb{P}^{1}$ mentioned in the beginning:

It is given a sub-bundle $\mathcal{E}$ of the constant bundle $\mathcal{O}_{C} \oplus \mathcal{O}_{C}(\infty)$, with quotient concentrated on $\{\infty\} \times \mathbb{P}^{1}$ and isomorphic to $\mathcal{O}(1)$. Thus the determinant of cohomology of $\mathcal{E}$ has degree -1 .

Finally we finish by showing that the restriction of $\mathcal{L}(P)$ to $S L_{2}$-torsors is isomorphic to the determinant of cohomology. For this we use that line-bundles are uniquely determined by their central charge, that is the second half of the theorem (which we show next, of course without using the following arguments). Thus the Picard-group is infinite cyclic, and it suffices if both line-bundles have the same non-zero degree on one curve. 
To construct such an example consider an $S L_{2}$ torsor on $C \times \mathbb{P}^{1}$ of the form $\mathcal{E}=\mathcal{M} \oplus \mathcal{M}^{\otimes-1}$, with $\mathcal{M}$ a very ample line-bundle on $C \times \mathbb{P}^{1}$. For its determinant of cohomology we obtain $-<\mathcal{M}, \mathcal{M}>$, whose degree is the negative selfintersection of $\mathcal{M}$ (and non-zero).

The computation of the degree of $\mathcal{L}(P)$ is more complicated. We know that the structure-group of $P$ reduces to $B$ (even to $T$ ), and thus by pullback from $X_{P}$ we obtain as before a candidate $\mathcal{L}(P)^{\text {naiv }}$. We do not yet know that it coincides with $\mathcal{L}(P)$ because our Borel will not be "generic". However we easily construct a covering $S^{\prime} \rightarrow S=\mathbb{P}^{1}$ as needed for the definition of $\mathcal{L}(P)$, as follows:

For each positive root $\alpha$ of $G$ the line-bundle $\mathcal{L}_{\alpha}$ is a positive power of $\mathcal{M}$. Its global sections (over $C \times \mathbb{P}^{1}$ ) are affine spaces, and the product of these affine spaces is isomorphic to the space of global sections of the unipotent $N_{P}^{-}$opposite to the unipotent radical of $B_{P}$. Conjugating $B_{P}$ by these sections defines the desired family of "generic" Borel's over the product $S^{\prime}$ of $S$ and this affine space.

Thus over $S^{\prime} \mathcal{L}(P)$ is given by our formula. Pulling back via the zero-section we derive that this also holds over $S$. Note that rigidification does not change this picture because $C$ is constant.

Now finally the map $S L_{2} \rightarrow G$ induces an embedding $\mathbb{P}^{1} \subseteq X$, and the pullback of $\mathcal{L}(\lambda)$ under it is $\mathcal{O}\left(\lambda\left(H_{\theta}\right)\right)$. It follows that $\mathcal{L}(P)$ becomes a multiple of $<\mathcal{M}, \mathcal{M}>$, where the factor is the negative of

$$
\begin{aligned}
& \sum_{i} a_{i} \epsilon_{i}\left(H_{\theta}\right)^{2}+\sum_{i<j} a_{i j} \epsilon_{i}\left(H_{\theta}\right) \epsilon_{i}\left(H_{\theta}\right) \\
= & 1 / 2 \sum_{i, j}<H_{\alpha_{i}}, H_{\alpha_{j}}>\cdot \epsilon_{i}\left(H_{\theta}\right) \epsilon_{i}\left(H_{\theta}\right) .
\end{aligned}
$$

But this quadratic form is $W$-invariant, so it takes the same value on $H_{\theta}$ as on $H_{\alpha_{i}}$, for any long simple root $\alpha_{i}$. And finally this value is 1 .

All in all we have finished the constructive part of the proof, and can pass to uniqueness.

Suppose a line-bundle $\mathcal{L}$ on $\mathcal{M}_{G}=R_{C^{0} / S}(G) \backslash \mathcal{D}_{G}$ has trivial central charge, that is comes from an operation of $R_{C^{0} / S}(G)$ on the trivial line-bundle on $\mathcal{D}_{G}$. It then corresponds to a character of $R_{C^{0} / S}(G)$, and we have to show that all such characters are trivial. We do this following an argument communicated to me by A. Beilinson, who (as I remember) attributes it to V.G. Drinfeld.

As we are working over a noetherian base and as $R_{C^{0} / S}(G)$ is inductive limit of algebraic schemes over this base, it suffices to show that the character is trivial on any point with values in an artinian local ring $R$, with algebraically closed residue-field $k$. We also may assume that our base is $S=\operatorname{Spec}(R)$.

For any finite set $T \subset C$ of $k$-points containing $\{\infty\}$ we may form the open affine subscheme $C-T \subset C$. As $G$-torsors are étale locally trivial on $C_{T}$ the moduli-stack $\mathcal{M}_{G}$ can be represented as

$$
\mathcal{M}_{G}=R_{C-T / S}(G) \backslash \prod_{x \in T} \mathcal{D}_{G, x} .
$$


The product is over copies of $\mathcal{D}_{G}$ indexed by $x \in T$, and one has to choose local parameters at all $x \in T$.

Thus $\mathcal{L}$ defines an $R_{C-T / S}(G)$-equivariant line-bundle on the product. The isomorphism class of this line-bundle (forgetting equivariance) is given by a tuple of central charges $c(x) \in \mathbb{Z}$. These do not change if we enlarge $T$, thus define a function from $C(k)$ to $\mathbb{Z}$, which extends to a linear function on divisors. I claim that this function vanishes on principal divisors:

This can be checked over the base $k$. Assume $\operatorname{div}(f)=\sum_{x \in T} n(x) x$ is a principal divisor. Define two homomorphisms $S L_{2} \rightarrow R_{C-T / S}(G)$ as follows: One is the constant homomorphism defined by the highest $\operatorname{root} \theta$, the other one is its twist by $f$ (with root-vectors $f X_{-\theta}, f^{-1} X_{\theta}$ ). They coincide on diagonal matrices, that is they share the same one-parameter group $H_{\theta}: \mathbb{G}_{m} \rightarrow R_{C-T / S}(G)$. However if we choose the unique lifts (of $S L_{2}$ 's) into the product of central extensions $\tilde{L} G_{x}$, the two characters differ by central characters $n(x)$, and the actions of the diagonal matrices on the fibre of $\mathcal{L}$ at the origine differ by $\sum_{x \in T} n(x) c(x)$. But these two actions are induced from the same action of $R_{C-T / S}(G)$, thus must coincide, and the sum vanishes.

So finally our map $c(x)$ factors over the Picard-group of $C$. As the Jacobian of $C$ is divisible it must be trivial on divisors of degree 0 . As it is also trivial on $\infty$ all $c(x)$ vanish.

It follows (now again over $R$ ) that $\mathcal{L}$ is defined by an action of $R_{C-T / S}(G)$ on the trivial bundle, that is by a character. That is the original character of $R_{C^{0} / S}(G)$ extends to all $R_{C-T / S}(G)$. It is necessarily trivial on the constants $G$, and also on all unipotents $R_{C-T / S}\left(\mathbb{G}_{a}\right)$ parametrised by roots, as these lie in the commutator.

To show that it is trivial on an arbitrary $g \in R_{C-T / S}(G)(R)$ we denote by $K$ the function-field of $C \otimes_{R} k$, and note that $G(K)$ is generated by its unipotent subgroups. Thus the image of $g$ in $G(K)$ can be written as the product of unipotent elements. These lie in some $R_{C-T^{\prime} / S}(G)(k)$ ( $T^{\prime}$ sufficiently big) and lift to $R_{C-T^{\prime} / S}(G)(R)$. Hence we may assume that $g$ reduces to 1 modulo the maximal ideal of $R$, that is it lies in the formal group. But by the usual infinitesimal arguments (as in Lemma $10 \mathrm{~b}$ )) this is contained in the subgroup generated by unipotents.

This finishes the proof of the theorem.

Remark. Everything works for twisted inner forms, and I think also for outer forms.

Remark. Suppose $P$ is a $G$-torsor in $C$. Then the center $Z(G)$ of $G$ acts trivially on the fibre of $\mathcal{L}$ in $P$. Namely $Z(G) \subset R_{C^{0} / S}(G)$ acts trivially on $\mathcal{D}_{G}$, so its action on the line-bundle $\mathcal{L}$ is given by a character. Considering the fibre in the origin one sees that this character extends to $G$, thus must be trivial. However other automorphisms may act non-trivially, even for stable torsors. This happens if the centralisers of elements of $G$ are not simply connected. The simplest example occurs for $\operatorname{Spin}(6)$ (where $\mathcal{L}$ is the Pfaffian). As a consequence $\mathcal{L}$ does not descend to a line-bundle on the coarse moduli-space of stable $G$ torsors. 


\section{References}

1. Beauville, A., Laszlo, Y.: Conformal blocks and generalized theta functions. Commun. Math. Phys. 164, 385-419 (1994)

2. Beauville, A., Laszlo, Y.: Un lemme de descente. C. R. Acad. Sci., Paris, Sér. I, Math. 320, 335-340 (1995)

3. Beauville, A., Laszlo, Y., Sorger, C.: The Picard group of the moduli of $G$-bundles on a curve. Comput. Math. 112, 183-216 (1998)

4. Deligne, P.: Le déterminant de la cohomologie. Contemp. Math. 67, 93-177 (1987)

5. Drinfeld, V., Simpson, C.: $B$-structures on $G$-bundles and local triviality. Math. Res. Lett. 2, 823-829 (1995)

6. Faltings, G.: A proof for the Verlinde formula. J. Algebr. Geom. 3, 347-374 (1994)

7. Kac, V.G.: Infinite dimensional Lie-algebras. Cambridge: Cambridge Univ. Press 1985

8. Kumar, S.: Demazure character formula in arbitrary Kac-Moody setting. Invent. Math. 89, 395-423 (1987)

9. Littelmann, P.: Contracting modules and standard monomial theory for symmetrizable Kac-Moody algebras. J. Am. Math. Soc. 11, 551-567 (1998)

10. Laszlo, Y., Sorger, C.: The line bundles on the moduli of parabolic $G$-bundles over curves and their sections. Ann. Sci. Éc. Norm. Supér., IV. Sér. 30, 499-525 (1997)

11. Mathieu, O.: Formules de caractères pour les algèbres de Kac-Moody générales. Astérisque 159/60 (1988)

12. Pappas, G., Rapoport, M.: Local models in the ramified case. I. The EL-case. math.AG/0006222

13. Ramanathan, A.: Schubert varieties are Cohen-macaulay. Invent. Math. 80, 283-294 (1985)

14. Sorger, C.: On moduli of $G$-bundles of a curve for exceptional $G$. Ann. Sci. Éc. Norm. Supér., IV. Sér. 32, 127-133 (1999)

15. Steinberg, R.: Regular elements of semisimple algebraic groups. Publ. Math., Inst. Hautes Etud. Sci. 25, 49-80 (1965)

16. Tsuchiya, A., Ueno, K., Yamada, Y.: Conformal field theories on universal families of stable curves with gauge symmetries. Adv. Stud. Pure Math. 19, 459-566 (1989), Princeton Univ. Press 